\title{
Preferences, entry, and market structure
}

\author{
Paolo Bertoletti* \\ and \\ Federico Etro**
}

We provide a unified approach to imperfect (monopolistic, Bertrand, and Cournot) competition when preferences are symmetric over a finite but endogenous number of goods. Markups depend on the Morishima elasticity of substitution and on the number of varieties. The comparative statics of free-entry equilibria is examined, establishing the conditions for markup neutrality with respect to income, market size, and productivity. We compare endogenous and optimal market structures for several non-CES examples. With a generalized linear direct utility, the markup can be constant and optimal under monopolistic competition, and nonmonotonic in the number of firms under Bertrand or Cournot competition.

\section{Introduction}

To understand how demand and supply fundamentals affect the structure of a market, namely, how many firms are active in it, how much they produce, and at which markup they sell, is a basic concern of economic theory. This important issue arises in the partial equilibrium analysis of industrial organization, as well as in general equilibrium applications, for instance, in international trade and macroeconomics. ${ }^{1}$ However, most of the modern theory of imperfect competition in markets with product differentiation is based on a few, specific microfoundations of the demand side. Models of monopolistic competition are usually based on CES preferences

*University of Pavia; paolo.bertoletti@unipv.it.

**University of Venice Ca' Foscari; federico.etro@unive.it.

We are very grateful to Giuseppe Savaré for fundamental clarifications on the properties of symmetric functions, but of course, the usual disclaimer applies. We also thank Giacomo Corneo, Paolo Epifani, Massimo Filippini, Atsushi Kajii, Giorgio Rampa, Lorenza Rossi, Ina Simonovska, Kresimir Zigic, two anonymous referees, and especially the Editor, Mark Armstrong, for useful comments. Seminar participants at the International Workshop on Global Competition and Innovation (Awaji, Japan, March 1, 2014), Free University of Berlin, Kyoto University, Yonsei University (Seoul), Padova University, Pavia University, University of Italian Swiss (Lugano), Delhi School of Economics, and the 2015 SAET conference at the University of Cambridge (UK) provided insightful comments. Further results can be found in the Working Paper version with the title, "A General Theory of Endogenous Market Structures" (DEM W.P. no. 81, University of Pavia, Dept. of Economics and Management, June 2014).

${ }^{1}$ There is a wide empirical literature pointing out that markups are affected by the number of consumers, their income, and productivity shocks. For recent examples, see Campbell and Hopenhayn (2005) on competition effects in industrial organization, Simonovska (2015) on pricing to market in trade, and Nekarda and Ramey (2013) on the cyclicality of markups in macroeconomics. 
or on separable direct utilities as in Dixit and Stiglitz (D-S, 1977) or on quasilinear preferences as in Spence (1976), and more recently Melitz and Ottaviano (2008), and Anderson, Erkal, and Piccinin (2012). ${ }^{2}$ As is well known, strategic interactions à la Cournot or Bertrand add a competitive element to the equilibrium of monopolistic competition, but also their analysis has been usually limited to a few microfounded examples. Other cases analyzed in the literature include those of homothetic preferences (Benassy, 1996; Feenstra, 2003 and 2014; D'Aspremont, Dos Santos Ferreira, and Gerard-Varet, 2007) and of separable indirect utilities (Bertoletti and Etro, 2014). Beyond these cases, little is known about how preferences shape competition and the incentives to enter a market.

In this work, we provide a first step to characterize, under a general microfoundation, the basic elements of an endogenous market structure: the number of active firms and the quantity/price strategies adopted by them. We endogenize consumer behavior, entry choices, and market strategies, but we take as a given the technological conditions and the mode of competition, which we assume to be either monopolistic competition à la Chamberlin, Bertrand competition in prices, or Cournot competition in quantities. Although this is typical of trade and macroeconomic applications, we leave for future research the extension to additional interesting aspects of firm behavior, such as the endogenous determination of technology through Research and Development (R\&D) activity (Sutton, 1991) and of the mode of competition (see D'Aspremont, Dos Santos Ferreira, and Gerard-Varet, 2007).

Our purpose is to analyze the three main forms of competition when consumers are endowed with general (nonseparable) preferences that are symmetric ${ }^{3}$ over a large number of differentiated goods, of which only some are endogenously provided. We start proposing a generalized definition of monopolistic competition that applies to all cases with a large but finite number of goods. Exploiting the properties of symmetric preferences, we show that the relevant demand elasticity is provided by the so-called Morishima elasticity of substitution (as defined in Blackorby and Russell, 1981), ${ }^{4}$ and can be computed directly from the utility function. We also show how the markups relevant in Bertrand and Cournot symmetric equilibria depend on the same elasticity and on the number of varieties actually provided. These results confirm that the difference between the true demand elasticity and that perceived by firms under our definition of monopolistic competition is indeed negligible when the number of goods is large enough. ${ }^{5}$ On this basis, we characterize the implications of free entry and the comparative statics of the associated endogenous market structure. To illustrate how to derive imperfect competition equilibria, we reconsider a variety of preferences used in the literature on monopolistic competition, such as additive preferences or examples of homothetic preferences. ${ }^{6}$ In addition, we provide the first characterization of the equilibria under the so-called generalized linear direct utility proposed by Diewert (1971), and under quadratic utilities that are neither homothetic nor additive. ${ }^{7}$ Following Feenstra (2003), we account for changes in the functional form representing preferences due to changes in the number of available goods.

\footnotetext{
${ }^{2}$ The case of additively separable preferences has been recently reconsidered by Zhelobodko et al. (2012) and Bertoletti and Epifani (2014). Many results are also known for quasilinear preferences with an outside good (Vives, 1999), but this assumption has the cost of eliminating any mechanism through which income directly affects demand.

${ }^{3}$ In the economics of product differentiation, the assumption of symmetric preferences is the hallmark of the “Chamberlinian paradigm," as first formalized by D-S (1977).

${ }^{4}$ Notice that in the general case of preferences defined over more than two goods, there are different ways of defining the elasticity of substitution (see Blackorby and Russell, 1989).

${ }^{5}$ This is known in models based on additive preferences: see, for instance, Yang and Heijdra (1993) and Bertoletti and Epifani (2014). We show that it holds in all models with symmetric preferences.

${ }^{6}$ In particular, we consider translog preferences and the so-called generalized Leontief preferences: these belong to the so-called "quadratic mean of order $r$ " (QMOR) expenditure functions recently reexamined by Feenstra (2014).

${ }^{7}$ Although we are not aware of an earlier use of the quadratic indirect utility considered here, the quadratic direct utility is derived by the quasilinear preferences of Melitz and Ottaviano (2008), eliminating the outside good and has been used elsewhere under monopolistic competition.
} 
Our investigation allows us to shed some light on a number of issues in the theory of imperfect competition. For instance, in the analysis of monopolistic competition, we provide a new example (under a generalized linear direct utility) of non-CES preferences that deliver constant equilibrium markups. This is indeed a case where the Morishima elasticity of substitution is fixed under symmetric consumption. Moreover, we show that homothetic preferences generate prices that could either decrease with more competitors, if their goods are perceived as becoming more substitutable, or increase when they are perceived as becoming more differentiated, and we have closed-form examples in both directions. In the analysis of strategic interactions, we generalize two results already established in the literature for quasilinear, homothetic, and additive preferences (see, respectively, Vives, 1999; D’Aspremont, Dos Santos Ferreira, and Gerard-Varet, 2007; Bertoletti and Epifani, 2014): the Cournot markup is higher than the Bertrand markup and both are higher than the monopolistic competition markup and converge to the latter when the number of firms becomes infinite. Moreover, we present novel examples in which Cournot and Bertrand markups initially decrease but subsequently increase in the number of firms.

A main contribution of our work is to characterize the comparative statics of markups under endogenous market structures, which may have applications for the modern theories of trade and macroeconomics featuring imperfect competition. In particular, we characterize the conditions on preferences under which in a well-behaved unique free-entry equilibrium either neutrality or competitive effects arise with respect to changes in three key parameters: (i) the number of consumers, which represents market size in trade models or labor force in macroeconomics; (ii) the individual income of consumers, whose changes correspond to cross-country income differences in trade, and to demand shocks in macroeconomics; and (iii) the marginal cost, whose reduction corresponds to globalization in trade and to a productivity boost in macroeconomics. Results are simple under monopolistic competition. The neutrality of income on markups holds under any additive direct utility and also with a quadratic indirect utility. The neutrality of population on markups holds not only with CES preferences, but also under any additive indirect utility, with all of its consequences in terms of pure gains from variety due to a larger market size. The neutrality of productivity on markups always holds under homothetic preferences, because only the number of firms affects them, so that changes in the marginal cost are translated proportionally on prices (complete pass-through) and never affect the number of firms. Additional competitive effects emerge in our analysis under Bertrand and Cournot competition. Investigating which of these potential market effects actually arises is left as an important task for empirical research.

Finally, we analyze the optimal allocations for general preferences and compare them with the endogenous market structures. To exemplify our results, we discuss examples with or without excess entry. Remarkably, under generalized linear preferences, we show that monopolistic competition generates insufficient (excess) entry if and only if markups are increasing (decreasing) in the number of firms, and efficiency when they are independent from the number of firms. This shows for the first time that efficiency of monopolistic competition equilibria can occur beyond the case of CES preferences.

The novelty of our contribution is to generalize results that have been (separately) established only for three classes of preferences (homothetic and directly or indirectly additive). Therefore, it is important to clarify which of our results are novel and their relation with the recent literature. The characterization of Chamberlinian, Bertrand, and Cournot symmetric equilibria through the simple derivation of the Morishima elasticity of substitution, which holds for all symmetric preferences, is established here for the first time, as well as the general conditions for the neutrality of income, market size, and productivity on markups. However, the neutrality of productivity under homotheticity is a simple consequence of the dependence of markups on the number of goods alone, known at least since Kimball (1995) and Benassy (1996), the neutrality of income under direct additivity was noticed by Zhelobodko et al. (2012), and the neutrality of market size under indirect additivity has been derived in Bertoletti and Etro (2016). We show that these neutralities 
can also emerge under more general conditions, and we provide new examples of preferences delivering constant markup or just income neutrality under monopolistic competition.

An independent work by Parenti, Ushchev, and Thisse (2014) has also analyzed monopolistic competition under a general microfoundation with a continuum of goods. This work does not analyze (given the continuum of goods) the Bertrand and Cournot equilibria and it does not identify the role of the Morishima elasticity of substitution. Moreover, it does not address the problem of dependence of symmetric preferences on the number of varieties not (yet) provided by the market. However, Parenti, Ushchev, and Thisse (2014) confirm the three neutralities for the main classes of preferences and independently derive rules for the optimal market structure. Their analysis can be seen as complementary to ours from a methodological point of view because it is done in the limit case of a continuum of goods. It is also useful because it allows for extensions to an outside commodity, heterogeneous consumers and heterogeneous firms, which can be exploited for general equilibrium applications. Trade models of monopolistic competition with a continuum of heterogeneous firms for homothetic and directly and indirectly additive preferences are presented also in Feenstra (2014), Arkolakis et al. (2015), and Bertoletti, Etro, and Simonovska (2016).

The article is organized as follows: Section 2 describes the general model for a given number of firms and Section 3 illustrates it in examples, Section 4 endogenizes the number of firms, and Section 5 discusses optimality. Section 6 discusses informally additional applications of our framework and concludes. Technical details are left to the Appendixes.

\section{The model}

Consider a market populated by $L$ identical consumers with income $E>0$ to be spent on a number of differentiated goods, each one produced by a firm with marginal cost $c$ and fixed cost $F$ (if labor is used as the numeraire, $c$ and $F$ can be measured in terms of units of labor and $E$ interpreted as the individual labor endowment). The price for variety $j$ is $p_{j}$ and its individual consumption is given by $x_{j}$.

We assume that the potential (arbitrarily large but finite) number of varieties, $N$, is given, and that preferences over them are symmetric (on the properties of symmetric preferences, see Appendix A). However, only a number $n<N$ of varieties are actually consumed in the market, and preferences can then be represented equivalently by symmetric direct or indirect utility functions as:

$$
U^{n}=U^{n}(\mathbf{x}) \quad \text { or } \quad V^{n}=V^{n}(\mathbf{s}),
$$

where $s_{i}=p_{i} / E, i=1, \ldots n$ are the normalized prices. To satisfy standard conditions, it is assumed that, for any $n, U^{n}(\cdot)$ is increasing and quasiconcave, that $V^{n}(\cdot)$ is decreasing and quasiconvex, and that both are (at least three times) differentiable. Notice that, as in Feenstra (2003), utility functions (1) are naturally indexed by the number $n$, and are endogenously derived from the underlying preferences (kept fixed), imposing that $N-n$ varieties have a price so high that their demands are nil (see Appendix B).

The inverse and direct (Marshallian) demand systems are immediately provided by the following FOCs for utility maximization and the Roy identities ${ }^{8}$ :

$$
\mathbf{s}(\mathbf{x})=\frac{D U^{n}(\mathbf{x})}{\widetilde{\mu}^{n}(\mathbf{x})}, \quad \mathbf{x}(\mathbf{s})=\frac{D V^{n}(\mathbf{s})}{\mu^{n}(\mathbf{s})},
$$

where

$$
\tilde{\mu}^{n}(\mathbf{x})=\sum_{j=1}^{n} U_{j}^{n}(\mathbf{x}) x_{j}, \quad \mu^{n}(\mathbf{s})=\sum_{j=1}^{n} V_{j}^{n}(\mathbf{s}) s_{j},
$$

\footnotetext{
${ }^{8}$ Throughout this article, we assume that $\mathbf{x}(\mathbf{s})$ as well as $\mathbf{s}(\mathbf{x})$ behave well, and in particular are unique. For the sake of simplicity, we omit the index $n$. 
and suffixes denote partial derivatives. Notice that $\left|\mu^{n}(\mathbf{s})\right|=\widetilde{\mu}^{n}(\mathbf{x}(\mathbf{s}))$ is the relevant marginal utility of income (multiplied by income $E$ ). It is now useful to state a fundamental property of symmetric preferences (the proof is provided in Appendix A):

Lemma 1. Symmetry of preferences implies that:

$$
U_{i}^{n}(\mathbf{x})=h^{n}\left(x_{i}, \mathbf{x}\right) \quad \text { and } \quad V_{i}^{n}(\mathbf{s})=g^{n}\left(s_{i}, \mathbf{s}\right),
$$

where $h^{n}$ and $g^{n}$ are symmetric functions with respect to $x$ and $s$, respectively, for given $x_{i}$ and $s_{i}$.

It follows that $\tilde{\mu}^{n}$ and $\mu^{n}$ are symmetric functions, too, and that the inverse and direct demand functions:

$$
s_{i}\left(x_{i}, \mathbf{x}\right)=\frac{h^{n}\left(x_{i}, \mathbf{x}\right)}{\widetilde{\mu}^{n}(\mathbf{x})}, \quad x_{i}\left(s_{i}, \mathbf{s}\right)=\frac{g^{n}\left(s_{i}, \mathbf{s}\right)}{\mu^{n}(\mathbf{s})},
$$

$i=1, \ldots, n$, are symmetric with respect to their second (vector) arguments.

The profits of firm $i$ can be written as:

$$
\pi_{i}=\left(p_{i}-c\right) x_{i} L-F .
$$

The equilibrium market structure depends on the form of competition, and we will examine separately three different forms. Monopolistic competition neglects strategic interactions and is useful to analyze markets with many firms (especially in general equilibrium applications), whereas Bertrand and Cournot competition take in consideration strategic interactions and are suitable to analyze markets with a small (and possibly endogenous) number of firms. First, we will focus on equilibrium pricing and production decisions for a given number of varieties/firms, and then we will characterize the endogenous market structures determined by free entry.

Monopolistic competition. Within a large market, each firm can reasonably hold the belief that its actions will have no major effect on rivals. This core assumption of monopolistic competition is obviously satisfied in models with a continuum of goods: in equilibrium firms, which cannot influence their competitors' profit, correctly anticipate the value of the market variables and compute their demand elasticity. When the number of goods is finite but sufficiently large and preferences are separable (namely, $h^{n}$ and/or $g^{n}$ depend only on the own choices), D-S (1977) suggested to model monopolistic competition by neglecting strategic interactions although still assuming that firms anticipate the value of the equilibrium demand shifter (namely, $\widetilde{\mu}^{n}$ and/or $\mu^{n}$ ). Intuitively, this is reasonable because the impact of individual choices on the shifter will indeed be negligible if the number of firms is large. However, we need a definition of this market structure which applies also to the case of general preferences. According to (5), the direct effect of a firm choice on its demand is just captured by the partial derivatives of demand with respect to its first argument:

$$
h_{1}^{n}\left(x_{i}, \mathbf{x}\right) \text { and } g_{1}^{n}\left(s_{i}, \mathbf{s}\right),
$$

whereas the indirect effect, analogous to the impact of a competitor's choice, operates through the symmetric component of demand. This suggests the following generalized definition of monopolistic competition, which extends the D-S (1977) proposal' ${ }^{9}$ :

Definition 1. Monopolistic competition characterizes a market structure in which each firm sets its choice ignoring its indirect impact on demand although correctly anticipating the value of the market variables.

\footnotetext{
${ }^{9}$ Notice that $h^{n}$ or $g^{n}$ might be independent, respectively, from $x_{i}$ and $s_{i}$ (given, respectively, $\mathbf{x}$ and $\mathbf{s}$ ). This is what happens in the case of homogeneous goods, where $h^{n}\left(\sum_{j=1}^{n} x_{j}\right)$ depends only on the total quantity consumed, leading to well-known results. Throughout this article, we assume on the contrary that $h^{n}$ and $g^{n}$ are monotonic with respect to their first arguments.
} 
Notice that in many applications, this is equivalent to say that each firm takes as given the value of some symmetric choice aggregators (and the number of competitors $n$ ). This happens not only under direct additivity of preferences (D-S, 1977) or indirect additivity (Bertoletti and Etro, 2014), but also whenever it is possible to define appropriate "market indexes," as with homothetic preferences or in the aggregative games studied by Anderson, Erkal, and Piccinin (2012). However, under nonseparable preferences, demands generally depend intrinsically on competitors' choices, and accordingly our definition extends the domain of application of monopolistic competition to all symmetric settings. ${ }^{10}$ Obviously, to be acceptable as a behavioral assumption it requires that the market is sufficiently large to make negligible the indirect effect discussed above.

As for monopolistic behavior, the profit maximizing choices are independent from the strategic variable adopted (price or quantity). To verify that this is the case, notice that inverting $h^{n}\left(x_{i}, \mathbf{x}\right)$ in (5) one obtains:

$$
x_{i}=\left(h^{n}\right)^{-1}\left(s_{i} \tilde{\mu}^{n}(\mathbf{x}(\mathbf{s})), \mathbf{x}(\mathbf{s})\right)=x_{i}\left(s_{i}, \mathbf{s}\right),
$$

that is, the partial inverse of $h^{n}$ with respect to its first argument gives the value of $x_{i}$. This implies that the perceived demand function under monopolistic competition does not depend on the variable (price or quantity) chosen to express firm's choice.

According to Definition 1, the perceived inverse demand elasticity and the perceived direct demand elasticity in monopolistic competition are given by:

$$
\epsilon^{n}\left(x_{i}, \mathbf{x}\right)=-\frac{h_{1}^{n}\left(x_{i}, \mathbf{x}\right) x_{i}}{h^{n}\left(x_{i}, \mathbf{x}\right)} \quad \text { and } \quad \varepsilon^{n}\left(s_{i}, \mathbf{s}\right)=-\frac{s_{i} g_{1}^{n}\left(s_{i}, \mathbf{s}\right)}{g^{n}\left(s_{i}, \mathbf{s}\right)},
$$

which are, of course, one the reciprocal of the other when evaluated at $\mathbf{x}(\mathbf{s})$. Standard conditions for the maximization of profits (6) show that $\epsilon^{n}\left(x_{i}, \mathbf{x}\right)$ and $\varepsilon^{n}\left(s_{i}, \mathbf{s}\right)$ determine the optimal pricing rules under monopolistic competition:

$$
\frac{p_{i}-c}{p_{i}}=\epsilon^{n}\left(x_{i}, \mathbf{x}\right)=\frac{1}{\varepsilon^{n}\left(s_{i}, \mathbf{s}\right)} .
$$

Such a condition must hold also when firms have different marginal costs, say $c_{i}$ for firm $i$, as often assumed in trade models since the work of Melitz (2003). ${ }^{11}$ However, in what follows, we focus on the case of firms with identical cost functions looking for symmetric equilibria, for which we simplify our notation to $\epsilon^{n}(x) \equiv \epsilon^{n}(x, x \iota)$ and $\varepsilon^{n}(s) \equiv \varepsilon^{n}(s, s \iota)$, where $\iota$ is the relevant unit vector. This allows us to obtain immediately:

Proposition 1. In any symmetric equilibrium of monopolistic competition:

$$
\frac{p_{-c}}{p}=\epsilon^{n}(x)=\frac{1}{\varepsilon^{n}(s)} .
$$

The explicit solution for the symmetric equilibrium price can be obtained using the budget constraint $n x s=1$. Notice that $(p-c) / p$ is a continuous, increasing function from $[c, \infty)$ onto $[0,1)$ : thus, a monopolistic competition equilibrium exists if $0<\epsilon^{n}(x)<1$ or $\varepsilon^{n}(s)>1$ everywhere. $^{12}$

The role of the Morishima elasticity. The interpretation of the elasticities in (7) is not immediate because we are departing from the common assumptions of separability. However,

\footnotetext{
${ }^{10}$ This definition actually suggests a way of generalizing our approach to the case of asymmetric preferences: see the cases of generalized additivity (Pollak, 1972) and implicit additivity (Hanoch, 1974).

${ }^{11}$ Although in this article we deal only with the case of symmetric firms, the reader interested in applications to the case of (a continuum of) heterogeneous firms can refer to Feenstra (2014), Parenti, Ushchev, and Thisse (2014), Arkolakis et al. (2015), and Bertoletti, Etro, and Simonovska (2016).

${ }^{12}$ Sufficient conditions for uniqueness of the equilibrium are obviously $\partial \varepsilon^{n}(s) / \partial s \geq 0$ and $\partial \epsilon^{n}(x) / \partial x \leq 0$ for any $s$ and $x$.
} 
we can provide both a constructive approach to characterize equilibria and an interpretation of our results by referring to the elasticity of substitution. The literature has developed alternative generalizations of the substitutability measure introduced by John Hicks and Joan Robinson for the case of two goods under homotheticity (see Hicks, 1970 and Blackorby and Russell, 1989). Here, we apply to the demand systems (5) the so-called Morishima Elasticity of Complementarity (MEC), $\epsilon_{i j}(\mathbf{x})$, and the Morishima Elasticity of Substitution (MES), $\varepsilon_{i j}(\mathbf{s})$, as defined in Blackorby and Russell (1981). These measures correspond to the elasticities of the price ratio $s_{i} / s_{j}$ with respect to the quantity of good $i, x_{i}$, and of the quantity ratio $x_{i} / x_{j}$ with respect to the price of $\operatorname{good} i, s_{i}($ for $i \neq j)$ :

$$
\epsilon_{i j} \equiv-\frac{\partial\left(s_{i} / s_{j}\right)}{\partial x_{i}} \frac{x_{i}}{\left(s_{i} / s_{j}\right)} \quad \text { and } \quad \varepsilon_{i j} \equiv-\frac{\partial\left(x_{i} / x_{j}\right)}{\partial s_{i}} \frac{s_{i}}{\left(x_{i} / x_{j}\right)},
$$

and can be directly computed for any preferences in (1) by deriving the price ratio $s_{i} / s_{j}=U_{i}^{n} / U_{j}^{n}$ and the quantity ratio $x_{i} / x_{j}=V_{i}^{n} / V_{j}^{n}$. To see their role in our setting, we need to rewrite the perceived demand elasticity. By symmetry of preferences, whenever $s_{i}=s_{j}$ and $x_{i}=x_{j}$ we have:

$$
h_{1}^{n}\left(x_{i}, \mathbf{x}\right)=U_{i i}^{n}(\mathbf{x})-U_{i j}^{n}(\mathbf{x}) \quad \text { and } \quad g_{1}^{n}\left(s_{i}, \mathbf{s}\right)=V_{i i}^{n}(\mathbf{s})-V_{i j}^{n}(\mathbf{s}) .
$$

Using this, by direct computation we can express the perceived demand elasticities (7) as follows:

$$
\epsilon^{n}=\frac{x_{i} U_{j i}^{n}}{U_{j}^{n}}-\frac{x_{i} U_{i i}^{n}}{U_{i}^{n}}=\epsilon_{i j} \text { and } \varepsilon^{n}=\frac{s_{i} V_{j i}^{n}}{V_{j}^{n}}-\frac{s_{i} V_{i i}^{n}}{V_{i}^{n}}=\varepsilon_{i j},
$$

where we used symmetry again to replace $U_{i}^{n}$ with $U_{j}^{n}$ and $V_{i}^{n}$ with $V_{j}^{n}$. Accordingly, the perceived demand elasticity that is relevant for firms active under monopolistic competition corresponds to the elasticity of the relative demand of two goods when their relative price changes (starting from identical prices). Moreover, given any preferences (1), one can directly compute the monopolistic competition equilibrium by calculating the MES or the MEC as shown above.

Of course, our result generalizes the role of the constant elasticity of substitution under CES preferences in shaping monopolistic markups. ${ }^{13}$ It provides a rationale for the well-known fact that, under direct additivity of preferences (D-S, 1977), the perceived demand elasticity depends only on the individual consumption of each variety and, in particular, on the elasticity of the marginal utility of consumption - what Zhelobodko et al. (2012) define as "relative love for variety." It also explains why under indirect additivity (Bertoletti and Etro, 2014) the perceived demand elasticity of each firm depends only on its price-income ratio. In addition, the result that with homothetic preferences the symmetric demand elasticity depends only on $n$ (Kimball, 1995; Benassy, 1996) follows from the homogeneity properties of the previous elasticities under homotheticity. Moreover, our formulas apply in the more general case of nonadditive and nonhomothetic preferences, opening up unexplored scenarios. This perspective also illuminates the relationship between the elasticities perceived under monopolistic competition and the demand elasticities relevant in case of strategic interactions, which are our next focus.

Cournot competition. We now move to the case of Nash equilibria which arise when firms compete by choosing how much to produce. Consider the following (standard) definition:

Definition 2. Cournot competition characterizes a market structure in which each firm sets its production level taking in consideration its total impact on the inverse demand and taking as given the production level of each other firm.

\footnotetext{
${ }^{13}$ In this article, we refer to "markup" either as the ratio $p / c$ or as the monotonically related expressions $p / c-1$ and $1-c / p$ (the latter is the so called "Lerner index"): obviously, they are determined by the relevant pricing rule based on the (perceived) demand elasticity.
} 
The relevant Cournotian "individual marginal revenue" of firm $i, M R_{i}=\partial\left\{p_{i} x_{i}\right\} / \partial x_{i}$, can be written as:

$$
\begin{aligned}
M R_{i} & =\frac{\left[U_{i}^{n}(\mathbf{x})+U_{i i}^{n}(\mathbf{x}) x_{i}\right] \mu^{n}(\mathbf{x})+U_{i}^{n}(\mathbf{x}) x_{i}\left[U_{i}^{n}(\mathbf{x})+\sum_{j=1}^{n} U_{j i}^{n}(\mathbf{x}) x_{j}\right]}{-\widetilde{\mu}^{n}(\mathbf{x})^{2} / E} \\
& =p_{i}(\mathbf{x})\left\{1-s_{i}(\mathbf{x}) x_{i}+\frac{U_{i i}^{n}(\mathbf{x}) x_{i}}{U_{i}^{n}(\mathbf{x})}-\sum_{j=1}^{n} \frac{U_{j i}^{n}(\mathbf{x}) x_{i}}{U_{j}^{n}(\mathbf{x})} s_{j}(\mathbf{x}) x_{j}\right\} \\
& =p_{i}(\mathbf{x})\left\{1-s_{i}(\mathbf{x}) x_{i}-\sum_{j \neq i}^{n} \epsilon_{i j}(\mathbf{x}) s_{j}(\mathbf{x}) x_{j}\right\} \\
& =p_{i}(\mathbf{x}) \sum_{j \neq i}^{n}\left[1-\epsilon_{i j}(\mathbf{x})\right] s_{j}(\mathbf{x}) x_{j},
\end{aligned}
$$

where $\epsilon_{i j}$ is again the MEC between varieties $i$ and $j$ and $s_{j} x_{j}$ is the market share of firm $j$. Notice that positive marginal revenues require that the elasticities of complementarity are "on average" (with market shares as weights) smaller than 1. Equating the marginal revenue to the marginal $\operatorname{cost} c$, in a Cournot equilibrium we have:

$$
\frac{p_{i}(\mathbf{x})-c}{p_{i}(\mathbf{x})}=s_{i}(\mathbf{x}) x_{i}+\sum_{j \neq i}^{n} \epsilon_{i j}(\mathbf{x}) s_{j}(\mathbf{x}) x_{j},
$$

which provides the general version of the Cournotian pricing rule.

Focusing on the case of a symmetric equilibrium, the markup becomes $1 / n+(n-$ 1) $\epsilon^{n}(x) / n$, which exhibits a simple relationship with the pricing rule of the monopolistic competition equilibrium. Using $\epsilon^{n}(x)=1 / \varepsilon^{n}(s)$, we can express the same rule in terms of the MESs drawn from the indirect utility. ${ }^{14}$ This proves the following result, where the superscript $C$ refers to Cournot:

Proposition 2. In any symmetric Cournot equilibrium:

$$
\frac{p^{C}-c}{p^{C}}=\frac{1}{n}+\frac{n-1}{n} \epsilon^{n}\left(x^{C}\right)=\frac{1}{n}+\frac{n-1}{n \varepsilon^{n}\left(s^{C}\right)} .
$$

One implication of this result is that one can directly compute the symmetric Cournot equilibrium for a given number of firms after deriving the appropriate Morishima elasticities from the primitive preferences: in the next section, we will provide similar computations for cases where a direct derivation of the Cournot equilibrium would be intractable. To compare monopolistic and Cournot competiton, notice that $1>\frac{1}{n}+\frac{n-1}{n} \epsilon^{n}(x)>\epsilon^{n}(x)$ if $\epsilon^{n}<1$. Accordingly, the assumption $\epsilon^{n}<1$ allows to conclude that the Cournot markup is always higher than the monopolistic competition markup for given preferences, a result already known for a variety of examples (as with quasilinear, separable, or homothetic preferences), but not in general. The ranking depends on the fact that Cournotian firms internalize also the indirect impact of their production level on marginal revenues, which is the same as the increase of production by any competitor: as goods are substitutes in a symmetric equilibrium, ${ }^{15}$ this impact is negative and internalizing it amounts to reduce production and increase markups. ${ }^{16}$

\footnotetext{
${ }^{14}$ An alternative proof can be provided by obtaining the elasticity of the direct demand from the inversion of the inverse demand system at its symmetric equilibrium.

${ }^{15}$ When demand is elastic (and there is no outside commodity), a price increase must raise the expenditure in the other commodities.

${ }^{16}$ More formally, let us differentiate $\sum_{j=1}^{n} s_{j}(\mathbf{x}) x_{j}=1$ with respect to $x_{i}$, making use of (5). In a symmetric equilibrium, this gives $1=-\sum_{k=1}^{n} \frac{\partial s_{k}}{\partial x_{i}} \frac{x}{s}=\epsilon^{n}-n \frac{\partial s_{j}}{\partial x_{i}} \frac{x}{s}(i \neq j)$. Then, $\epsilon^{n}<1$ implies that $\partial s_{j} / \partial x_{i}<0$, that is, that varieties are "gross, $q$-substitutes" (see Hicks, 1970, for this terminology) in a symmetric equilibrium. Accordingly, 
Bertrand competition. Let us now consider the Nash equilibria in the case firms use price strategies. We adopt the following (standard) definition:

Definition 3. Bertrand competition characterizes a market structure in which each firm sets its price taking in consideration its total impact on the direct demand and taking as given the price of each other firm.

Bertrand firms set their Lerner index as the inverse of the true elasticity of their Marshallian direct demand (5). This is:

$$
\begin{aligned}
\left|\frac{\partial \ln x_{i}}{\partial \ln p_{i}}\right| & =-\frac{s_{i}}{x_{i}(\mathbf{s})} \frac{V_{i i}^{n}(\mathbf{s}) \mu^{n}(\mathbf{s})-V_{i}^{n}(\mathbf{s})\left[V_{i}^{n}(\mathbf{s})+\sum_{j=1}^{n} V_{j i}^{n}(\mathbf{s}) s_{j}\right]}{\left[\mu^{n}(\mathbf{s})\right]^{2}} \\
& =-\frac{s_{i} V_{i i}^{n}(\mathbf{s})}{V_{i}^{n}(\mathbf{s})}+\frac{s_{i}}{\mu^{n}(\mathbf{s})}\left[V_{i}^{n}(\mathbf{s})+V_{i i}^{n}(\mathbf{s}) s_{i}+\sum_{j \neq i}^{n} V_{j i}^{n}(\mathbf{s}) s_{j}\right] \\
& =s_{i} x_{i}(\mathbf{s})+\sum_{j \neq i}^{n}\left[\frac{s_{i} V_{j i}^{n}(\mathbf{s})}{V_{j}^{n}(\mathbf{s})}-\frac{s_{i} V_{i i}^{n}(\mathbf{s})}{V_{i}^{n}(\mathbf{s})}\right] s_{j} x_{j}(\mathbf{s}) \\
& =1+\sum_{j \neq i}^{n}\left(\varepsilon_{i j}(\mathbf{s})-1\right) s_{j} x_{j}(\mathbf{s}),
\end{aligned}
$$

which depends on the MESs with the other varieties. Note that, if $s_{i}=s$ (and then $x_{i}=x$ ), $i=1, \ldots, n$ :

$$
\left|\frac{\partial \ln x_{i}}{\partial \ln p_{i}}\right|=\frac{n-1}{n} \varepsilon^{n}+\frac{1}{n},
$$

confirming the expected result for which the difference between the true demand elasticity and that perceived by firms under monopolistic competition is negligible when $n$ is large. Even when the consumption pattern is not symmetric (e.g., because neither preferences are), by manipulating the Slutsky equations one can easily prove that:

$$
\left|\frac{\partial \ln x_{i}}{\partial \ln p_{i}}\right|=\varepsilon_{i j}-\frac{\partial \ln \tilde{x}_{j}}{\partial \ln p_{i}}+s_{i} x_{i} \frac{\partial \ln x_{i}}{\partial \ln E},
$$

where $\tilde{x}_{j}$ is the compensated (Hicksian) demand for variety $j$. As $\sum s_{i} x_{i} \frac{\partial \ln x_{i}}{\partial \ln E}=1$, the last term is necessary small when $n$ is large if variety $i$ does not play a special role, and the previous expression says that the MES is approximately equal to demand elasticity when cross demand effects (here, measured by the symmetric term $\partial \ln \tilde{x}_{j} / \partial \ln p_{i}$ ) are indeed negligible. Finally, notice that in the CES case, the elasticity above is the same of Yang and Heijdra (1993), whose equilibrium, therefore, corresponds to a Bertrand equilibrium.

To satisfy the FOCs for profit maximization, it is necessary that "on average" (by using the market shares as weights) the MESs are larger than 1. In a symmetric Bertrand equilibrium, markups simplify to the inverse of the true elasticity, and using $\epsilon^{n}(x)=1 / \varepsilon^{n}(s)$, we reach the following result, where the superscript $B$ refers to Bertrand:

Proposition 3. In any symmetric Bertrand equilibrium:

$$
\frac{p^{B}-c}{p^{B}}=\frac{n}{1+(n-1) \varepsilon^{n}\left(s^{B}\right)}=\frac{n \epsilon^{n}\left(x^{B}\right)}{\epsilon^{n}\left(x^{B}\right)+n-1} .
$$

Cournotian firms face an elasticity of the inverse demand $\epsilon^{n}-\frac{\partial s_{j}}{\partial x_{i}} \frac{x}{s}$, which is larger than the one perceived by firms in monopolistic competition, and set a higher markup. 
Again, this allows one to directly compute the symmetric Bertrand equilibrium simply deriving the appropriate MES or MEC from the utility function, as we will see in next section. Notice that $\left[1+(n-1) \varepsilon^{n}(s)\right] / n>\varepsilon^{n}(s)$ if $\varepsilon^{n}>1$. Thus, the assumption $\varepsilon^{n}>1$ makes rankable the equilibrium markups in Bertrand and monopolistic competition. ${ }^{17}$ In line with a classic result first derived by Vives (1985) under quasilinear preferences, the Bertrand markup must be below the Cournot markup. Therefore, we can summarize the ranking of markups as follows:

Corollary 1. Assume $\varepsilon^{n}>1\left(\epsilon^{n}<1\right)$ everywhere: then for any Cournot equilibrium markup, there exists a lower Bertrand equilibrium markup which in turn is larger than a monopolistic competition equilibrium markup.

Finally, we can generalize results already established for particular preferences (see Vives, 1999; D’Aspremont, Dos Santos Ferreira, and Gerard-Varet, 2007; Bertoletti and Epifani, 2014) about the convergence of Bertrand and Cournot equilibria when the number of firms increases indefinitely:

Corollary 2. The Cournot and Bertrand equilibrium markups converge to the monopolistic competition markups if the number of firms goes to infinity.

Of course, it is only when the goods tend to become perfect substitutes (the MES increases unbounded) that all the three equilibria converge to perfect competition.

\section{Old and new examples}

In this section, we review particular cases of our general setting, and propose some new applications with closed-form solutions. is: ${ }^{18}$

Directly additive preferences. D-S (1977) assume direct additivity of preferences, that

$$
U^{n}(\mathbf{x})=\sum_{j=1}^{n} u\left(x_{j}\right)
$$

with $u^{\prime \prime}<0<u^{\prime}$ for $x>0$. Such a functional form does not actually change with $n$ (provided that $u(0)=0$ ), and implies that $U_{j i}^{n}=0$ : the MEC is then $\epsilon_{i j}=-x_{i} u^{\prime \prime}\left(x_{i}\right) / u^{\prime}\left(x_{i}\right)=\epsilon\left(x_{i}\right)$, which depends neither on the behavior nor on the number of competitors (conditionally on $x_{i}$ ). The equilibrium prices under monopolistic competition, Bertrand competition, and Cournot competition can be derived, respectively, as:

$$
p=\frac{c}{1-\epsilon(x)}, \quad p^{B}=\frac{\left[\epsilon\left(x^{B}\right)+(n-1)\right] c}{(n-1)\left(1-\epsilon\left(x^{B}\right)\right)}, \quad \text { and } \quad p^{C}=\frac{n c}{(n-1)\left(1-\epsilon\left(x^{C}\right)\right)},
$$

where the budget constraint can be used to explicitly solve these relations. The equilibrium markup under monopolistic competition is decreasing or increasing in the number of firms depending on whether $\epsilon^{\prime}(x)$ is positive or negative (because entry reduces the consumption of individual varieties). For instance, the (negative) exponential case $u=1-e^{-x}$, first analyzed in

${ }^{17}$ The intuition is again simple and relies on the internalization of the indirect impact of a price increase on demand under Bertrand competition. Differentiating $\sum_{j} s_{j} x_{j}(\mathbf{s})=1$ with respect to $s_{i}$ and making use of (5) yields that, in a symmetric equilibrium $1=-\sum_{k=1}^{n} \frac{\partial x_{k}}{\partial s_{i}} \frac{s}{x}=\varepsilon^{n}-n \frac{\partial x_{j}}{\partial s_{i}} \frac{s}{x}(i \neq j)$. Then, $\varepsilon^{n}>1$ implies that $\frac{\partial x_{j}}{\partial s_{i}}>0$, that is, that varieties are "gross, $p$-substitutes" (Hicks, 1970). Accordingly, Bertrand firms perceive the true Marshallian elasticity $\epsilon^{n}-\frac{\partial x_{j}}{\partial s_{i}} \frac{s}{x}$, which is smaller than the one perceived by firms in monopolistic competition, and actually price higher.

${ }^{18} \mathrm{D}-\mathrm{S}$ (1977) also considered an outside commodity with consumed quantity $x^{\circ}$ : extending our definition of monopolistic competition to such a setting (i.e., with utility functions $\widetilde{U}\left(x^{o}, U^{n}(\mathbf{x})\right)$ and $\tilde{V}\left(s^{o}, V^{n}(\mathbf{s})\right)$, where $s^{o}$ is the price-income ratio of the outside good) is straightforward. For an example with indirect additivity, see Bertoletti and Etro (2014). On quasilinear preferences, see Vives (1999) and Anderson, Erkal, and Piccinin (2012). 
Bertoletti (1998), delivers $\epsilon=x$, therefore, the markup declines with entry. In particular, the three equilibrium prices can be derived as:

$$
p=c+\frac{E}{n}, \quad p^{B}=\frac{E+n c}{2 n}\left[1+\sqrt{1+\frac{4 n c E}{(n-1)(E+n c)^{2}}}\right], \quad \text { and } \quad p^{C}=\frac{n c}{n-1}+\frac{E}{n} .
$$

Instead, a first example of markups increasing in the number of firms was provided by Bertoletti, Fumagalli, and Poletti (2008) for subutilities such as $u=x+2 \sqrt{x}$, where $\epsilon=1 /(2+2 \sqrt{x})$. $^{19}$ Strategic interactions determine markups that can be nonmonotonic in the number of firms.

Indirectly additive preferences. Bertoletti and Etro (2014) assume indirect additivity, that is:

$$
V^{n}(\mathbf{s})=\sum_{j=1}^{n} v\left(s_{j}\right)
$$

with $v^{\prime \prime}>0>v^{\prime}$. Such a functional form again does not change with $n$ (if $v=0$ for the varieties that are not purchased) and implies $V_{j i}^{n}=0$ : the MES is $\varepsilon_{i j}=-s_{i} v^{\prime \prime}\left(s_{i}\right) / v^{\prime}\left(s_{i}\right)=\varepsilon\left(s_{i}\right)$, which again depends neither on the competitors' behavior nor on their number. The three symmetric equilibria can be derived as:

$$
p=\frac{\varepsilon(s) c}{\varepsilon(s)-1}, \quad p^{B}=\frac{\left[1+(n-1) \varepsilon\left(s^{B}\right)\right] c}{(n-1)\left(\varepsilon\left(s^{B}\right)-1\right)}, \quad \text { and } \quad p^{C}=\frac{n \varepsilon\left(s^{C}\right) c}{(n-1)\left(\varepsilon\left(s^{C}\right)-1\right)} .
$$

Markups are independent from the number of firms under monopolistic competition and decreasing with respect to $n$ under strategic interactions. Our formulas allow a direct computation. With the "addilog" ${ }^{20}$ specification $v=\frac{(a-s)^{1+\gamma}}{1+\gamma}$, where $a>0$ and $\gamma>0$ parametrize willingness to pay and demand elasticity, we have $\varepsilon=\gamma s /(a-s)$ and the equilibrium prices are:

$$
p=\frac{a E+\gamma c}{\gamma+1}, p^{B}=\frac{\Gamma+\sqrt{\Gamma^{2}+\frac{4 a E c(\gamma+1)}{n+1}}}{2(\gamma+1)}, \quad \text { and } \quad p^{C}=\frac{a E+\frac{n \gamma c}{n-1}}{\gamma+1},
$$

where $\Gamma=a E+\gamma c-c /(n-1)$. The (negative) exponential case $v=e^{-b s}$, with $b>0$, provides $\varepsilon=b s$ and the equilibrium prices:

$$
p=c+\frac{E}{b}, \quad p^{B}=\frac{1}{2}\left[\Lambda+\sqrt{\Lambda^{2}+\frac{4 c E}{(n+1) b}}\right], \quad \text { and } \quad p^{C}=\frac{n c}{n-1}+\frac{E}{b},
$$

where $\Lambda=c+E / b$.

Homothetic preferences. Benassy (1996) discussed some instances of homothetic preferences: as it is well known that the elasticities $\epsilon_{i j}$ and $\varepsilon_{i j}$ are homogeneous of degree zero under homotheticity, the symmetric equilibrium markups must be a function of the number of varieties alone. Here, we will present three non-CES ${ }^{21}$ examples in detail.

${ }^{19}$ Notice that the CES case emerges in the isoelastic case: $u=x^{\rho} / \rho, \rho<1$. Bertoletti, Fumagalli, and Poletti (2008) studied the case of the "mixtures" $u=x^{\rho} / \rho+x^{\delta} / \delta, 1 \geq \rho>\delta>0$, which generate a decreasing $\epsilon(x)$.

${ }^{20}$ Bertoletti and Etro (2014) propose this name as a tribute to Houthakker (1960), who used this terminology for additive specifications.

${ }^{21}$ The classes of (symmetric) directly additive, indirectly additive, and homothetic symmetric preferences have only a common member, given by the CES preferences. Notice that in endogenous growth models à la Romer (1990) with multiple inputs produced by monopolistic firms, one can replace a CES technology with any constant returns to scale technology and obtain markups which depend on the number of inputs alone. 
Translog preferences. Feenstra (2003) has shown that, taking into account the endogenous choice of the goods to be consumed, symmetric translog preferences imply:

$$
V^{n}(\mathbf{s})=\exp \left[-a_{0}-\frac{1}{n} \sum_{j=1}^{n} \log s_{j}-\frac{1}{2} \sum_{k=1}^{n} \sum_{j=1}^{n} a_{k j} \log s_{k} \log s_{j}\right],
$$

where $a_{0}=1 /(2 \gamma n)-1 /(2 \gamma N), a_{k j}=\gamma / n>0$ for $k, j=1, \ldots, n, k \neq j$, and $a_{j j}=-\gamma(n-$ $1) / n$, with $\gamma>0$. In a symmetric equilibrium, we can directly compute $\varepsilon^{n}=1+\gamma n$, and the three equilibria can be derived as follows:

$$
p=\left(1+\frac{1}{\gamma n}\right) c, \quad p^{B}=\left(1+\frac{1}{\gamma(n-1)}\right) c, \quad \text { and } \quad p^{C}=\frac{1+\gamma n}{\gamma(n-1)} c .
$$

Markups are decreasing in the number of goods and would become negligible when $n \rightarrow N$ if the number of potential varieties $N$ is large enough.

Generalized Leontief preferences. As in the case of translog preferences, the generalized Leontief preferences, originally presented in Diewert (1971) and recently considered by Feenstra (2014) in a setting with monopolistic competition, can be represented by a so-called flexible functional form (see Varian, 1992). Over a set of $N$ potential goods, this is provided by the following indirect utility function:

$$
V=[\sqrt{\mathbf{s}} \mathbf{B} \sqrt{\mathbf{s}}]^{-1}
$$

where $\sqrt{\mathbf{s}^{\prime}}=\left[\sqrt{s_{1}}, \ldots, \sqrt{s_{N}}\right]$ is defined as the (row) vector of the square roots of the normalized prices, and $\mathbf{B}$ is a $N \times N$ matrix. To achieve symmetry and satisfy regularity assumptions, we set $b_{j j}=b<0$ and, without loss of generality, $b_{k j}=1$ for $k, j=1, \ldots, N, k \neq j$. After some tedious algebra, it can be shown that, assuming $b \in(-N+1,-N+2)$, preferences depend on the number of consumed varieties as follows:

$$
V^{n}(\mathbf{s})=\left[\sum_{k=1}^{n} \sum_{j=1}^{n} a_{k j} \sqrt{s_{k} s_{j}}\right]^{-1},
$$

with parameters $a_{i i}=\underline{a}<0$ and $a_{i j}=a>0$ for $i, j=1, \ldots, n, i \neq j$ defined in Appendix B as functions of $b, n$, and $N$.

Accordingly, we obtain the following demand for good $i$ :

$$
x_{i}=\frac{\underline{a}-a+a \sum_{k=1}^{n} \sqrt{\frac{s_{k}}{s_{i}}}}{\sum_{k=1}^{n} \sum_{j=1}^{n} a_{k j} \sqrt{s_{k} S_{j}}} .
$$

Because the perceived demand elasticity is:

$$
\varepsilon^{n}\left(s_{i}, \mathbf{s}\right)=\frac{\frac{a}{2} \sum_{k=1}^{n} \sqrt{\frac{s_{k}}{s_{i}}}}{\underline{a}-a+a \sum_{k=1}^{n} \sqrt{\frac{s_{k}}{s_{i}}}},
$$

in a symmetric equilibrium, we have:

$$
\varepsilon^{n}=\frac{n a}{2[\underline{a}+(n-1) a]}=\frac{n}{2(N+b-1)},
$$

which is linear in the number of consumed goods. This allows us to directly compute the three symmetric equilibria. Let us restrict attention to a specific case by setting $b=3 / 2-N$. Then, $\varepsilon^{n}=n$ and thus:

$$
p=\frac{n c}{n-1}, \quad p^{B}=\frac{\left(n^{2}-n+1\right) c}{(n-1)^{2}}, \text { and } p^{C}=\frac{n^{2} c}{(n-1)^{2}} .
$$


Again, markups decrease with the number of varieties and become negligible when $n \rightarrow N$ if $N$ is large enough.

Generalized linear direct utility. An example of interesting preferences that have not yet ${ }^{22}$ been applied to the analysis of imperfect competition can be represented by the following direct utility function (see Diewert, 1971, who associated it to the names of Roy Allen, Daniel McFadden, and Paul Samuelson and called these preferences generalized linear):

$$
U=\sqrt{\mathbf{x}} \mathbf{B} \sqrt{\mathbf{x}}
$$

where $\sqrt{\mathbf{x}^{\prime}}=\left[\sqrt{x_{1}}, . ., \sqrt{x_{N}}\right]$, the off-diagonal elements of the $N \times N$ matrix $\mathbf{B}, b_{i j}$, are positive and equal, and $b_{i i}=b(i, j=1, \ldots, N, i \neq j)$. Notice that $U$ is concave, homogeneous of degree 1 , and strictly monotonic if also $b>0$, which we assume.

If only $n<N$ varieties are actually available, this utility reduces to:

$$
U^{n}(\mathbf{x})=\sum_{i=1}^{n} \sum_{j=1}^{n} b_{i j} \sqrt{x_{i} x_{j}}
$$

whose functional form once again does not depend on $n$. Let us normalize $b_{i j}=1$ for any $i \neq j$, so that utility becomes $U=n x(n-1+b)$ in the case of symmetric consumption. Computation shows that $\tilde{\mu}^{n}(\mathbf{x})=U^{n}(\mathbf{x})$ by homogeneity, and as:

$$
h^{n}\left(x_{i}, \mathbf{x}\right)=b-1+\frac{1}{\sqrt{x_{i}}} \sum_{j=1}^{n} \sqrt{x_{j}}, \text { and } h_{1}^{n}\left(x_{i}, \mathbf{x}\right)=-\frac{x_{i}^{-3 / 2}}{2} \sum_{j=1}^{n} \sqrt{x_{j}},
$$

we obtain:

$$
\epsilon^{n}\left(x_{i}, \mathbf{x}\right)=\frac{\sum_{j=1}^{n} \sqrt{x_{j}}}{2 \sum_{j=1}^{n} b_{i j} \sqrt{x_{j}}} .
$$

Notice that in a symmetric equilibrium:

$$
\epsilon^{n}=\frac{n}{2(n-1+b)}
$$

which satisfies $\partial \epsilon^{n} / \partial n<0$ if and only if $b<1$.

An increase of $n$ produces an anticompetitive effect under monopolistic competition if $b>1$, but in any case we have $\lim _{n \rightarrow \infty} \epsilon^{n}=1 / 2$, that is, the markup would never become negligible even if $n$ was very large. In the specification with $b=1$, the equilibrium markup is independent from the number of goods because the MEC is constant for a symmetric consumption. Whereas CES preferences are the only separable preferences that induce constant markups, our result shows that there are other well-behaved preferences that produce the same result in symmetric equilibria, and further examples can be constructed. As usual, we can derive the full set of imperfect competition equilibrium prices:

$$
p=2 c, \quad p^{B}=\frac{(2 n-1) c}{n-1}, \quad \text { and } \quad p^{C}=\frac{2 n c}{n-1} .
$$

Also, the general case $(b \neq 1)$ provides interesting results, with markups that can increase in the number of firms not only under monopolistic competition, but also with strategic interactions. Under monopolistic competition, the general markup is:

$$
\frac{p-c}{p}=\frac{n}{2(n-1+b)}
$$

${ }^{22}$ It is worth noting that the generalized linear preferences, that can be represented by a direct utility function which is a quadratic mean of order 1, do not belong to the QMOR expenditure function class of preferences considered by Feenstra (2014). 
which is increasing in the number of firms if $b>1$. Strategic interactions add a competition effect, that however does not necessarily compensate the anticompetitive effect inherited through $\epsilon^{n}$. Indeed, under Cournot and Bertrand competition, we can compute the following markups:

$$
\frac{p^{C}-c}{p^{C}}=\frac{n^{2}+n-2+2 b}{2 n^{2}-2 n+2 n b} \quad \text { and } \quad \frac{p^{B}-c}{p^{B}}=\frac{n^{2}}{2 n^{2}-3 n+2+2(n-1) b},
$$

which might be both increasing in the number of firms if this is large enough. In particular, under Cournot competition, the markup is increasing with respect to $n$ if and only if:

$$
(2 n+1)\left[n^{2}-n+n b\right]>\left[n^{2}+n-2+2 b\right][n-1+b],
$$

which simplifies to $(b-2) n^{2}>4 n(b-1)+2 b^{2}-4 b+2$. Under Bertrand competition, the markup increases with respect to $n$ if:

$$
2 n\left[2 n^{2}-3 n+2+2(n-1) b\right]>n^{2}[4 n-3+2 b],
$$

that is, $n(2 b-3)>4(b-1)$. For instance, for $b=3$, the price is increasing in the number of firms if $n>4+\sqrt{24}$ under Cournot competition and if $n>8 / 3$ under Bertrand competition. As far as we know, this is the first computable example of Bertrand and Cournot equilibria with prices increasing in the number of firms in a representative consumer model (for similar results, but in spatial models, see Chen and Riordan, 2007). Notice, however, that even when the price is increasing in the number of firms, equilibrium profits remain decreasing in it, which will allow us to analyze endogenous market structures later on.

Quadratic direct utility. We now move to the general field of nonhomothetic preferences that are also nonseparable. Let us consider the following utility function:

$$
U^{n}(\mathbf{x})=\alpha \sum_{j=1}^{n} x_{j}-\frac{\gamma}{2} \sum_{j=1}^{n} x_{j}^{2}-\frac{\eta}{2}\left(\sum_{j=1}^{n} x_{j}\right)^{2},
$$

where $\alpha, \gamma, \eta>0$. This looks similar to the quasilinear utility function popularized by Melitz and Ottaviano (2008), but of course it differs from that because we do not have quasilinear preferences here (and we consider a finite number of goods). ${ }^{23}$ The functional form of $U^{n}(\mathbf{x})$ does not change with the number $n$ of available varieties, and its derivatives are given by $h^{n}\left(x_{i}, \mathbf{x}\right)=\alpha-\gamma x_{i}-$ $\eta \sum_{j=1}^{n} x_{j}$. Accordingly, the demand function perceived under monopolistic competition is a linear demand with $h_{1}^{n}\left(x_{i}, \mathbf{x}\right)=-\gamma$. It is easy to show that $U^{n}(\mathbf{x})$ is concave, and that if we restrict our attention to the case in which $h^{n}\left(x_{i}, \mathbf{x}\right)>0$ for any $i=1, \ldots, n$, it is also strictly monotonic. The inverse demand function for variety $i$ is given by:

$$
s_{i}=\frac{\alpha-\gamma x_{i}-\eta \sum_{j=1}^{n} x_{j}}{\alpha \sum_{j=1}^{n} x_{j}-\gamma \sum_{j=1}^{n} x_{j}^{2}-\eta\left(\sum_{j=1}^{n} x_{j}\right)^{2}},
$$

with perceived demand elasticity:

$$
\epsilon^{n}\left(x_{i}, \mathbf{x}\right)=\frac{\gamma x_{i}}{h^{n}\left(x_{i}, \mathbf{x}\right)}
$$

In the symmetric equilibrium, we obtain:

$$
\epsilon^{n}(x)=\frac{\gamma x}{\alpha-\gamma x-n \eta x}
$$

\footnotetext{
${ }^{23}$ For a recent example of a model with monopolistic competition and quasilinear quadratic preferences (and a continuum of goods), see Di Comite, Thisse, and Vandenbussche (2014).
} 
with $\partial \epsilon^{n} / \partial n, \partial \epsilon^{n} / \partial x>0, \lim _{x \rightarrow 0} \epsilon^{n}(x)=0$, and $\lim _{x \rightarrow \alpha /(2 \gamma+n \eta)} \epsilon^{n}(x)=1 .^{24}$ Therefore, we have:

$$
p=\frac{c}{1-\epsilon^{n}(x)}=m(x, n) c,
$$

where $m(x, n)=h^{n}(x) /(\alpha-2 \gamma x-n \eta x), m(0, n)=1$, and $\partial \ln m / \partial \ln x>\partial \ln m / \partial \ln n>0$. Because a monopolistic competition equilibrium is then characterized by:

$$
\frac{E}{n}=m(x, n) x c,
$$

we can be sure that such an equilibrium does exist and it is unique. Notice that (35) implies $\partial x / \partial E>0$ and $\partial x / \partial n<0$, and accordingly, $\partial p / \partial E>0$ and $\partial p / \partial n<0$ : a seemingly ${ }^{25}$ competitive effect emerges even in the absence of strategic interactions. Similarly, we obtain the following prices:

$$
p^{C}=\frac{n m\left(x^{C}, n\right) c}{(n-1)} \quad \text { and } \quad p^{B}=\frac{\left[n m\left(x^{B}, n\right)-1\right] c}{(n-1)}
$$

under Cournot and Bertrand competition.

Quadratic indirect utility. We finally introduce a class of generalized quadratic preferences that has not been explored (as far as we know) in the literature on imperfect competition:

$$
V=\mathbf{s}^{\prime} \boldsymbol{\beta} \mathbf{s}-\boldsymbol{\alpha}^{\prime} \mathbf{s},
$$

where $\boldsymbol{\beta}$ is a $N \times N$ matrix and $\boldsymbol{\alpha}$ a $N$ vector, and $N$ is, as usual, the given total number of potential varieties. To achieve symmetry and to obtain a well-behaved indirect utility function, we assume that $\alpha_{j}=\alpha>0, \beta_{i j}=-b<0$, and $\beta_{i i}=\beta, i, j=1, \ldots, N, i \neq j$. Normalizing $\beta=b(N-1)>0$, Appendix B shows that $V$ is convex $(\boldsymbol{\beta}$ is a positive semidefinite matrix $)$, and monotonic decreasing in the case $\alpha \boldsymbol{\iota} \geq 2 \boldsymbol{\beta}$ s, to which we restrict our attention. Moreover, after some tedious algebra, preferences over the $n$ consumed varieties can be expressed as:

$$
\begin{aligned}
V^{n}(\mathbf{s}) & =\sum_{j=1}^{n}\left[b(N-1) s_{j}^{2}-b s_{j} \sum_{k \neq j}^{n} s_{k}\right]-\alpha N-n b(N-n) \bar{s}^{2}-\frac{N-n}{n b} \frac{\alpha^{2}}{4} \\
& =\text { const }-\alpha N \bar{s}+n b[N \operatorname{Var}\{\mathbf{s}\}-n \operatorname{Cov}\{\mathbf{s}\}],
\end{aligned}
$$

where const $=-\frac{\alpha^{2}(N-n)}{4 n b}$ and $\bar{s}=\sum_{j=1}^{n} s_{j} / n$ is the mean value, $\operatorname{Var}\{\mathbf{s}\}=\sum_{j=1}^{n} s_{j}^{2} / n-\bar{s}^{2}$ the variance, and $\operatorname{Cov}\{\mathbf{s}\}=\sum_{j=1}^{n} \sum_{k=1}^{n} s_{j} s_{k} / n^{2}-\bar{s}^{2}$ the covariance of the normalized prices $\mathbf{s}$.

The individual demand function has thus the linear form:

$$
x_{i}=\frac{\frac{\alpha N}{n}-2 b N\left(s_{i}-\bar{s}\right)}{\left|\mu^{n}(\mathbf{s})\right|} .
$$

It is perhaps remarkable that, when we properly take into account the choke price of the unavailable varieties, the direct demand is perceived as linear in the difference between the own price and the average price of the available varieties, so reproducing the typical form adopted in textbook examples of monopolistic competition.

Because $g^{n}\left(s_{i}, \mathbf{s}\right)=2 b N\left(s_{i}-\bar{s}\right)-\alpha N / n$, the demand elasticity perceived in monopolistic competition is given by:

$$
\varepsilon^{n}\left(s_{i}, \mathbf{s}\right)=\frac{2 b s_{i}}{\frac{\alpha}{n}-2 b\left(s_{i}-\bar{s}\right)},
$$

${ }^{24}$ Accordingly, $\epsilon^{n}<1$ requires $x<\alpha /(2 \gamma+n \eta)$. Incidentally, notice that $\gamma \rightarrow 0$ leads to marginal-cost pricing (as goods tend to become perfect substitutes, monopolistic competition tends to perfect competition) and $\eta=0$ makes preferences directly additive.

${ }^{25}$ Notice that demand elasticity decreases with respect to $n$ for a given level of consumption $x$. However, it is easily proved that $d m / d n=\partial m / \partial n+(\partial m / \partial x)(\partial x / \partial n)<0$ if and ony if $\partial \ln m / \partial \ln x>\partial \ln m / \partial \ln n$, a condition which is here satisfied. 
which becomes

$$
\varepsilon^{n}(s)=\frac{2 b n s}{\alpha}
$$

in a symmetric equilibrium. ${ }^{26}$ Notice that $\varepsilon^{n}$ increases with $n s,{ }^{27}$ and solving for the symmetric equilibrium prices, we have:

$$
p=c+\frac{\alpha E}{2 b n}, \quad p^{B}=\frac{\Phi+\sqrt[2]{\Phi^{2}+\frac{8 n c b \alpha E}{n-1}}}{4 n b}, \quad \text { and } \quad p^{C}=\frac{n c}{(n-1)}+\frac{\alpha E}{2 b n},
$$

where $\Phi \equiv \alpha E+2 n c b$. Prices increase in income and decrease in the number of goods in all cases.

\section{Endogenous market structures}

We now consider the impact of free entry of firms. Our aim is to analyze how the main exogenous parameters, namely, the size of the market $L$, the income of consumers $E$, and the marginal cost of production $c$ affect the endogenous market structure, that is, the equilibrium number of firms, the price, and the production of each firm..$^{28}$

The symmetric equilibrium price must satisfies the condition:

$$
\frac{p-c}{p}=\frac{1}{\theta(p / E, n)},
$$

where the specific functional form for demand elasticity $\theta(s, n)>1$ depends on the mode of competition-see (8), (13), and (15). The zero-profit condition $(p-c) E L / p n=F$ provides the second equilibrium relation between the price and the number of firms:

$$
p=\frac{c E L}{E L-n F},
$$

and this is an increasing and convex function with respect to $n$, independent from the competition mode. Of course, the free-entry equilibrium is at the crossing of the two relations in the space $(n, p)$.

Let us define $\psi_{s}(s, n) \equiv \partial \ln \theta / \partial \ln s$ and $\psi_{n}(s, n) \equiv \partial \ln \theta / \partial \ln n$ as the partial elasticities of $\theta(s, n)$ with respect to price and number of firms, ${ }^{29}$ and let $A(s, n)=\theta-1+\psi_{s}$ and $D(s, n)=$ $(\theta-1)\left(1+\psi_{n}\right)+\psi_{s}$. We now show that $A, D>0$ are necessary and sufficient conditions for the existence of a unique and well-behaved (stable) equilibrium. The equilibrium profit as a function of the number of firms is

$$
\pi(n)=\frac{E L}{n \theta(s(n), n)}-F,
$$

where $s(n)$ solves (41), and by differentiation we obtain:

$$
\frac{d \ln s(n)}{d \ln n}=\frac{-\psi_{n}(s, n)}{A(s, n)} .
$$

Notice that the range of $\pi(n)$ is $(-F, \infty)$ : thus, a free-entry equilibrium with $\pi(n)=0$ exists and is unique for any $F, L, E, c>0$ if and only if $\pi^{\prime}(n)<0$ everywhere. Because:

$$
\frac{d\{n \theta(s(n), n)\}}{d n}=\theta(s, n)\left[1+\psi_{n}+\psi_{s} \frac{d \ln s}{d \ln n}\right]
$$

\footnotetext{
${ }^{26}$ Note that a comparison with (33) confirms that (38) and (32) actually represent different preferences.

${ }^{27}$ Under our assumptions, $\varepsilon^{n}>1$ requires $n s>\alpha / 2 b$.

${ }^{28}$ As it will become clear below from the equilibrium conditions, $L$ and $F$ have just opposite impacts on them.

${ }^{29}$ As usual in this literature, we treat $n$ as a continuous variable and assume the differentiability of the relevant functions with respect to $n$.
} 


$$
=\theta(s, n) \frac{D(s, n)}{A(s, n)},
$$

it follows that a unique free-entry equilibrium exists if and only if $D / A>0$ everywhere. Moreover, we can write the equilibrium system as:

$$
p-\frac{\theta(p / E, n)}{[\theta(p / E, n)-1]} c=0 \quad \text { and } \quad n-\frac{E L}{F \theta(p / E, n)}=0
$$

whose Jacobian is:

$$
J(p, n)=\left[\begin{array}{cc}
1+\frac{\psi_{s}}{\theta^{-1}} & \frac{p}{n} \psi_{n} \\
\frac{n}{p} \psi_{s} & 1+\psi_{n}
\end{array}\right] .
$$

According to a well-known result, the system (44) is then (locally) stable if and only if the real part of each eigenvalue of $-J$ is negative, a condition which is easily seen to be equivalent to $D>0 .{ }^{30}$ Thus, the necessary and sufficient condition for the existence of a unique and wellbehaved free-entry equilibrium is that $A$ and $D$ are both positive everywhere. ${ }^{31}$

Our first conclusion from these technicalities is a ranking of the equilibrium number of firms and markups emerging under the three forms of competition, as an immediate consequence of Corollary 1 and of the positive relation between price and number of firms noted above:

Proposition 4. Assume $A, D>0$ everywhere: the free-entry equilibrium number of firms and markup under Cournot competition are higher than those under Bertrand competition, which are higher than those under monopolistic competition.

The impact of market size, income, and productivity on the endogenous market structure. Total differentiation of the equilibrium system (44) provides the following elasticities of the equilibrium variables with respect to income $E$, market size $L$, and marginal $\operatorname{cost} c$ :

$$
\begin{aligned}
& \frac{d \ln p}{d \ln L}=\frac{-\psi_{n}}{D} \quad \text { and } \quad \frac{d \ln n}{d \ln L}=\frac{\theta-1+\psi_{s}}{D}>0 \\
& \frac{d \ln p}{d \ln E}=\frac{\psi_{s}-\psi_{n}}{D} \quad \text { and } \quad \frac{d \ln n}{d \ln E}=\frac{\theta-1+\theta \psi_{s}}{D} \\
& \frac{d \ln p}{d \ln c}=1-\frac{\psi_{s}}{D}>0 \quad \text { and } \quad \frac{d \ln n}{d \ln c}=\frac{-(\theta-1) \psi_{s}}{D},
\end{aligned}
$$

where the signs follows from our assumption that $A, D>0$. Let us call "markup neutrality" the property for which a change in one of the exogenous parameters does not affect the equilibrium markup. The comparative statics in (45)-(47) implies that the market size is neutral on the markup if and only if $\psi_{n}=0$, income is neutral if and only if $\psi_{s}=\psi_{n}$, and the marginal cost is neutral if and only if $\psi_{s}=0$. The implications for our examples are immediate under monopolistic competition:

(i) Market-size neutrality holds not only with CES preferences (Krugman, 1980) but under any case of indirect additivity: in these cases, a market that is twice as large attracts a double number of firms selling the same quantity at the same price and generating pure gains from variety $^{32}$;

\footnotetext{
${ }^{30}$ The condition is given by $\operatorname{tr}\{J\}>0$ and $|J|>0$.

${ }^{31}$ The use of the stability condition to give a sign to $A$ is, of course, an application of Samuelson's Correspondence Principle.

${ }^{32}$ Obviously, the equilibrium price is relative to the nominal price of the numeraire: that is, $\partial \ln p / \partial \ln E+$ $\partial \ln p / \partial \ln F+\partial \ln p / \partial \ln c=1$. Accordingly, different neutralities imply different empirical predictions that are in 
(ii) Income neutrality holds under direct additivity (see, e.g., Zhelobodko et al., 2012 and Bertoletti and Etro, 2014) and even in the quadratic indirect utility case because the elasticity of substitution depends on $n s$ under symmetric consumption: in these cases, a doubling of income induces a doubling of the number of firms;

(iii) Marginal-cost neutrality holds under all homothetic preferences, so that changes in productivity are translated proportionally on prices, as already noted in Kimball (1995) and Benassy (1996), and do not affect the number of firms: in trade and macroeconomic applications, this produces complete pass-through and markups that depend only on the number of firms.

The three neutralities hold at the same time, that is, markups are constant under CES preferences but also in other specifications generating a constant elasticity of substitution under symmetric consumption, as in our example of generalized linear preferences (with $b=1$ ).

Following a terminology which is widespread but overlooks the distinction between the role of the number of varieties in affecting substitutability (demand side) and the role of the number of competitors in shaping strategic interactions (supply side), we might say that an increase of income, market size, or productivity exerts a competition effect if it reduces the equilibrium markup. Notice that such an effect is associated in the first two cases (larger income and population) with an increase less than proportional of the number of firms, and in the third case (higher productivity) with a reduction of it (a case of so-called business destruction). The next result summarizes the general comparative statics of the free-entry equilibrium:

Proposition 5. Assume $A, D>0$ everywhere: (a) an increase in the market size exerts a competition effect if and only if $\psi_{n}>0$; (b) an increase in income exerts a competition effect if and only if $\psi_{s}<\psi_{n}$; (c) an increase in productivity exerts a competition effect if and only if $\psi_{s}<0$.

The implications for the case of monopolistic competition are again particularly revealing. To exemplify our results, let us consider the example of a quadratic direct utility, in which none of the mentioned three neutralities holds. From (33), we can obtain $\theta=n(\alpha s-\eta) / \gamma-1$, which implies: ${ }^{33}$

$$
\psi_{s}=\frac{\alpha s n}{\alpha s n-\eta n-\gamma}>\psi_{n}=\frac{n(\alpha s-\eta)}{n(\alpha s-\eta)-\gamma}>0 .
$$

Accordingly, under free entry, an increase in market size reduces markups $\left(\frac{d \ln p}{d \ln L}<0\right)$, an increase in productivity increases the markups $\left(\frac{d \ln p}{d \ln c}<1\right)$, and an increase in income increases prices $\left(\frac{d \ln p}{d \ln E}>0\right)$, expanding the number of firms more than proportionally.

Similar applications of the first result of Proposition 5 can be done to our other examples. In particular, let us focus first on the impact of an increase in market size: this issue is of particular relevance in trade theory because equivalent to opening up to frictionless trade (with an identical country), which determines welfare effects through increased variety and/or price changes (Krugman, 1979, 1980). It produces a competition effect under direct additivity when the elasticity of substitution decreases with the size of consumption, whereas an anticompetitive effect arises in the opposite case (see Zhelobodko et al., 2012 and Bertoletti and Epifani, 2014). A competition effect shows up also in the translog case, under generalized Leontief preferences and in the case of a quadratic indirect utility. Finally, a generalized linear direct utility can generate either a competitive or an anticompetitive effect of the market size.

The second result of Proposition 5 is about changes in income, that are particularly relevant for comparisons between countries with different per-capita income, or in macroeconomic analysis of the impact of demand shocks affecting consumer expenditure. A rise of income has

principle testable. For instance, indirect additivity implies $d \ln p / d \ln E+d \ln p / d \ln c=1$ : population should not affect prices and the elasticities of prices with respect to income and marginal cost should add to one.

${ }^{33}$ Notice that $\theta>1$ requires $n(\alpha s-\eta)>2 \gamma$. 
a competitive effect only if $\psi_{n}>\psi_{s}$ : clearly, this is a condition which can be satisfied under several cases, such as indirect additivity with $\psi_{s}<0$, or homotheticity with $\psi_{n}>0$ (a condition that holds with translog and generalized Leontief preferences). Nevertheless, markups increasing in income are much more in line with the trade evidence on pricing to market (for instance, see Simonovska, 2015 and Bertoletti, Etro, and Simonovska, 2016), and with some macroeconomic evidence on procyclical markups (Nekarda and Ramey, 2013): such a positive relation emerges naturally under indirect additivity when demand elasticity increases in the price-income ratio $\left(\psi_{s}>0\right)$.

The third result of Proposition 5 is about changes in the marginal cost, whose implications are critical in the macroeconomic analysis of the impact of supply shocks affecting productivity or, in other fields, such as public economics, when taxes are shifted on prices. Our result shows that the price sensitivity of demand elasticity is what matters to determine the translation of marginal costs (including taxes or exchange rates or any other cost wedge) on prices. In the (arguably) more realistic case in which demand is perceived as more rigid when income increases $\left(\psi_{s}>0\right)$, changes in productivity are translated less than proportionally on prices, and additional costs are undershifted (a case of incomplete pass-through). This is, for instance, what happens in both of our quadratic examples.

Moving briefly to the case of oligopolistic competition, strategic interactions tend to increase the value of $\psi_{n}$, thus raising the chances of getting competition effects due to an increase of market size or income. Consider, for instance, a population increase: even when a larger market size decreases demand elasticity under monopolistic competition, strategic interactions may give rise to a competition effect on markups, in particular if the number of firms is small. We can state unambiguous results with homothetic preferences, as in this case markups depend only on the number of goods:

Corollary 3. Under homotheticity, endogenous market structures generate neutrality of productivity, whereas income and market size exert a competition effect if and only if $\psi_{n}>0$.

The case of homothetic preferences is particularly relevant in dynamic (macroeconomic) applications. For example, Bilbiie, Ghironi, and Melitz (2012) have studied the impact of aggregate shocks in a monopolistic competition model with endogenous entry. These shocks can be magnified by competition effects when demand elasticity increases with entry, as with the translog preferences adopted by Bilbiie, Ghironi, and Melitz (2012) or with the introduction of strategic interactions à la Bertrand or Cournot, as in Etro and Colciago (2010).

Examples with monopolistic competition. We conclude this section with a few closedform solutions for the number of firms which can be used in applications, and are also useful for the welfare comparisons of the next section. We restrict the analysis to the case of monopolistic competition for the sake of brevity.

For the class of directly additive preferences, examples of free-entry equilibria with monopolistic competition are well known by now. Limiting our attention to the two examples mentioned in Section 3, the negative exponential case delivers the number of firms $n=\frac{E}{2 c}\left(\sqrt{1+\frac{c L}{F}}-1\right)$, whereas the example of Bertoletti, Fumagalli, and Poletti (2008) generates the equilibrium number of firms $n=\frac{E L}{2 F}\left[1+\frac{F}{C L}\left(1+\sqrt{1+\frac{C L}{F}}\right)\right]^{-1}$. It is easy to verify that an increase in population exerts a competition effect in the former case and an anticompetition effect in the latter.

For the class of indirectly additive preferences, the addilog specification delivers $n=\frac{(a E-c) E L}{F(a E+\gamma c)}$ firms under free entry, whereas the negative exponential gives $n=\frac{E^{2} L}{F(b c+E)}$ firms: both values are proportional to the population, but increase more than proportionally with an income expansion. Incidentally, notice that a more elastic demand (respectively, with higher values of the parameters $\gamma$ and $b$ ) reduces markups and the equilibrium number of firms. 
Let us move to homothetic preferences. In the translog example, we can solve for the free-entry number of goods as:

$$
n=\sqrt{\frac{E L}{\gamma F}+\left(\frac{1}{2 \gamma}\right)^{2}}-\frac{1}{2 \gamma} .
$$

This implies that total income $E L$ raises less than proportionally the number of firms: the reason is that business creation reduces the markups. Similar competition effects are associated to the case of the generalized Leontief preferences $(b=-N+3 / 2)$ :

$$
n=\sqrt{\frac{E L}{F}} .
$$

Instead, the case of a generalized linear direct utility delivers the following equilibrium under monopolistic competition:

$$
n=\frac{E L}{2 F}+1-b
$$

which is exactly proportional to total income $E L$ only if $b=1$ (and indeed, $\psi_{n}=0$ ). Otherwise, different outcomes are possible because larger and/or richer markets can either increase or decrease the markups.

Finally, let us consider our examples of nonhomothetic and nonseparable preferences. In the case of a quadratic direct utility, the closed-form solution is complex but we have already discussed its comparative statics. Under the quadratic indirect utility, the equilibrium number of firms with monopolistic competition is given by:

$$
n=\frac{\alpha E\left(\sqrt{1+\frac{8 b c L}{\alpha F}}-1\right)}{4 b c},
$$

which shows how the endogenous market structure is affected by all parameters: the number of firms/goods provided by the market increases proportionally with per-capita income but less than proportionally with market size, whereas an increase in productivity attracts entry and reduces markups. Most important, replacing the number of firms in the pricing rule (40), one can immediately verify that the free-entry equilibrium price is independent from income: accordingly, the neutrality of spending holds also under a quadratic indirect utility.

\section{Optimal market structures}

The optimal organization of production can be reached in our framework by a social planner controlling number of firms, production, and prices. ${ }^{34}$ Such an optimal market structure can be identified maximizing utility under the resource constraint $E L=n c x L+n F$. This is equivalent to the use of the consumer budget constraint and of the firm zero-profit condition, which can be combined to obtain (42). Let us define $U(x ; n)=U^{n}(x \iota)$ and equivalently, $V(s ; n)=V^{n}(s \iota)$. We can now define welfare as:

$$
W(n)=U\left(\frac{E}{c n}-\frac{F}{c L} ; n\right) \quad \text { or } \quad W(n)=V\left(\frac{c L}{E L-F n} ; n\right) .
$$

Let us also define $\phi(x ; n) \equiv U_{x}(x ; n) x / U(x ; n)$ as the elasticity of the direct utility with respect to consumption and $\varphi(x ; n) \equiv U_{n}(x ; n) n / U(x ; n)$ as the elasticity of the direct utility with respect to the number of varieties (notice that $\phi$ is the so-called "scale elasticity" of $U^{n}$ at $x \iota$ ). Similarly, let $\widetilde{\phi}(s ; n) \equiv-V_{s}(s ; n) s / V(s ; n)$ and $\widetilde{\varphi}(s ; n)=V_{n}(s ; n) n / V(s ; n)$.

\footnotetext{
${ }^{34}$ On the optimal market structures, see the original contribution by D-S (1977) and the extensions in Vives (1999), and more recently Mrázová and Neary (2014).
} 
Differentiating the equality $U(E / p n ; n)=V(p / E ; n)$, we obtain that $\phi(E / p n ; n)=\widetilde{\phi}(s ; n)$ and $\varphi(E / p n ; n)=\widetilde{\varphi}(s ; n)+\widetilde{\phi}(s ; n)$. We can now state the conditions for the optimal provision of goods in the following result:

Proposition 6. The optimal market structure requires

$$
p^{*}=c \frac{\varphi}{\phi} \quad \text { and } \quad n^{*}=\frac{E L(1-\phi / \varphi)}{F},
$$

where $\phi$ is the elasticity of direct utility with respect to the consumption of each good and $\varphi$ the elasticity of direct utility with respect to the number of varieties.

Rule (53) implies that price and number of goods provided should be increasing in $\varphi$, due to larger benefits from variety, and decreasing in $\phi$, due to larger benefits of expanding the consumption of each good.

The optimal market structure in case of direct additivity $(\varphi=1)$ was originally studied by D$\mathrm{S}$ (1977), and here we only illustrate it with our two examples. The exponential case $u=1-e^{-x}$ leads to excess entry, whereas the subutility $u=x+2 \sqrt{x}$, instead, leads to suboptimal entry under monopolistic competition. ${ }^{35}$ Indeed, in the former case, the optimal number of firms can be derived as:

$$
n^{*}=\frac{E}{c\left[-W_{-1}\left(-e^{-\frac{F}{c L}-1}\right)-1\right]},
$$

where $W_{-1}(\cdot)$ is the branch of the Lambert function satisfying $W_{-1} \leq-1$ (see Behrens and Murata, 2012), and in the latter case it is:

$$
n^{*}=\frac{E L}{2 F}\left[\frac{F}{4 c L}\left(1+\sqrt{1+\frac{4 c L}{F}}\right)+1\right]^{-1} .
$$

The optimal market structure under indirect additivity $(\widetilde{\varphi}=1)$ has been studied by Bertoletti and Etro (2014). Under addilog preferences $v=\frac{(a-s)^{1+\gamma}}{1+\gamma}$, the optimal number of firms can be derived as:

$$
n^{*}=\frac{E L(\Sigma-(2+\gamma) c)}{F(\Sigma+\gamma c)}
$$

where $\Sigma \equiv \sqrt{4(1+\gamma) a E+(\gamma c)^{2}}$. In the exponential case $v=e^{-b s}$, optimality requires:

$$
n^{*}=\frac{E L(\Xi-1)}{F(\Xi+1)}
$$

where $\Xi \equiv \sqrt{1+4 E / b c}$. It is easy to verify that in both cases, market equilibria involve excess entry. $^{36}$

Homothetic preferences have been analyzed by Benassy (1996), noticing the possibility of either excess or insufficient free entry under monopolistic competition. Indeed, under homotheticity, the scale of consumption does not matter for the optimal choice, and normalizing the direct utility to be homogeneous of degree 1 we get $U(x ; n)=x U^{n}(\iota)=n t(n) x$, with $\phi=1$ and $\varphi(n)=1+\chi(n)$, where $\chi(n)=d \ln t(n) / d \ln n$ is what Benassy (1996) called the "taste for

${ }^{35}$ This is so because the elasticities $\phi=u^{\prime}(x) x / u(x)$, which depend only on the consumption size $x$, are, respectively, decreasing and increasing in it (see D-S, 1977).

${ }^{36}$ This is due to the fact that the elasticities $\widetilde{\phi}=-v^{\prime}(s) s / v(s)$, which depend only on the normalized price $s$, are increasing with respect to it (see Bertoletti and Etro, 2014). 
variety" embedded into preferences. It follows from (53) and (44) that a sufficient condition for excess (insufficient) entry under homothetic preferences is $\chi(n)<(>) 1 /(\theta(n)-1)$. Analogously, we can write $V(s ; n)=V^{n}(\iota) / s=v(n) / s$, with $\widetilde{\varphi}(n)=d \ln v(n) / d \ln n$, and the sufficient condition for excess (insufficient) entry becomes $\widetilde{\varphi}(n)<(>) 1 /(\theta(n)-1)$.

In the case of translog preferences we actually have:

$$
V(s ; n)=\frac{1}{s} \exp \left(\frac{1}{2 \gamma N}-\frac{1}{2 \gamma n}\right)
$$

with

$$
\widetilde{\varphi}(n)=\frac{1}{2 \gamma n}<\frac{1}{\gamma n}=\frac{1}{\varepsilon^{n}-1} .
$$

This shows that the number of firms in a free-entry equilibrium is characterized by excess entry, as can be verified comparing $n$ from (48) to its optimal value:

$$
n^{*}=\sqrt{\frac{E L}{2 \gamma F}+\left(\frac{1}{4 \gamma}\right)^{2}}-\frac{1}{4 \gamma} .
$$

In the case of generalized Leontief preferences, always under our convenient specification $(b=-N+3 / 2)$, we obtain the following expression for welfare:

$$
V(s ; n)=\frac{2(2 n-1)}{\operatorname{sn}(2 N-1)},
$$

where

$$
\tilde{\varphi}(n)=\frac{1}{2 n-1}<\frac{1}{n-1}=\frac{1}{\varepsilon^{n}-1}
$$

shows that again excess entry occurs with imperfect competition. Indeed, the optimal number can be computed as:

$$
n^{*}=\sqrt{\frac{E L}{2 F}}
$$

A generalized linear direct utility implies:

$$
U(x ; n)=n x(n-1+b) .
$$

Because in this case:

$$
\chi(n)=\frac{n}{n-1+b}<\frac{n}{n-2+2 b}=\frac{\epsilon^{n}}{1-\epsilon^{n}},
$$

excess entry occurs under monopolistic competition when (perceived) demand elasticity is increasing in the number of firms $(b<1)$ and insufficient entry arises in the opposite case $(b>1)$. Indeed, the optimal number of firms can be computed as:

$$
n^{*}=\frac{E L}{2 F}+\frac{1-b}{2} \text {. }
$$

As expected, the equilibrium is efficient only in the notable case of a constant markup, that is, with $b=1$. As far as we know, this is the first example showing that monopolistic competition equilibria can be efficient beyond the case of CES preferences (of course, the corresponding strategic equilibria exhibit excess entry). 
Finally, let us move to preferences that are neither additive nor homothetic. In the case of a quadratic direct utility, we have $U(x ; n)=n x\left[\alpha-\frac{x}{2}(\gamma+\eta n)\right]$, and entry is generally inefficient. ${ }^{37}$ In the case of a quadratic indirect utility, we can derive:

$$
V(s ; n)=-\frac{N-n}{n b} \frac{\alpha^{2}}{4}-\alpha N s
$$

whose maximization delivers:

$$
n^{*}=\frac{E L}{F\left(1+2 \sqrt{\frac{b c L}{\alpha F}}\right)},
$$

which implies excess entry for all free-entry equilibria with imperfect competition. As these examples show, a variety of outcomes can emerge under general preferences because of the complex interplay of the elasticities of utility with respect to the consumption of each good and with respect to the number of varieties.

\section{Conclusion}

In this work, we have investigated how the nature of consumer preferences can shape entry and firm behavior in a market with imperfect competition. For this purpose, we have considered the technology to be common across firms and exogenous, and we have focused on the basic forms of competition (in prices or quantities), abstracting from other kinds of strategic interaction. The aim of this conclusive section is to discuss informally some of the possible extensions and applications, and to put future research into perspective.

Technology can be endogenized assuming that the fixed cost is an R\&D investment chosen by firms to reduce the marginal cost of production, as in the pioneering work of Dasgupta and Stiglitz (1980). In such a case, the optimal investment for each firm increases in the production level (Vives, 2008), and the symmetric equilibrium with free entry can be easily characterized. Under monopolistic competition, the classes of directly additive preferences and indirectly additive preferences preserve their respective "neutralities": only market size (income) affects prices and investments in the first (second) case. Therefore, when income (market size) increases, the number of firms changes linearly under directly (indirectly) additive preferences. Under strategic interactions, entry strengthens competition so that the endogenous number of firms can depend in more complex ways on the size of the market. For instance, the endogenous number of firms can be constant as in the Cournot model of Dasgupta and Stiglitz (1980), or exhibit an upper bound or a nonmonotonic relation with the size of the market in the spirit of Sutton (1991). A variety of examples are examined in Vives (2008), but a more general microfoundation requires further investigation.

Precommitments to additional strategic aspects or to the mode of competition can also be analyzed under additive preferences: the profit functions then depend on a single aggregator of quantities or prices, which is just the marginal utility of income. In this class of "aggregative games," we know (see, for instance, Etro, 2006 and Anderson, Erkal, and Piccinin, 2012) that, under free entry, any firm able to precommit acts with the purpose of becoming more aggressive than the others (i.e., of selling more at lower prices). For instance, a Stackelberg leader could precommit to set the monopolistic competition price, overinvest in cost reductions, adopt contractual agreements (with managers, banks, or other stakeholders) that incentivize aggressive pricing, or simply commit to compete in prices (rather than in quantities) to be more aggressive. As shown in Etro (2006), this is the only way to obtain positive profits in spite of free entry, gaining market shares, and reducing the number of entrants. Similar results hold also when multiple firms adopt simultaneously such precommitments, which suggests a general pattern. However, it would be

\footnotetext{
${ }^{37}$ See Nocco, Ottaviano, and Salto (2014) on a related analysis with quasilinear preferences and heterogeneous firms. 
useful to examine more general settings where all firms can commit to a preliminary strategy or to the mode of competition.

Last but not least, we want to stress some useful applications of our setting with general preferences to international trade and macroeconomics. The modern trade literature (Melitz, 2003 and Melitz and Ottaviano, 2008) has focused on general equilibrium models with heterogeneous firms, monopolistic competition, and free entry. Indeed, a stream of recent investigations have been proposed in multicountry "gravity" models with variable trade costs and a Pareto distribution of productivities, focusing on the three main classes of preferences: i.e., homothetic preferences in Feenstra (2014), directly additive preferences in Arkolakis et al. (2015), and indirectly additive preferences in Bertoletti, Etro, and Simonovska (2016). Remarkably, three different "neutralities" (with respect to income, population, and trade costs) emerge in these environments: under direct additivity, the distribution of consumption is the same across countries, under indirect additivity the distribution of (normalized) prices is the same across countries, and, finally, under homothetic preferences, the number of consumed goods is constant in each country. This resonates well with our results, supporting the idea that the nature of preferences of consumers is fundamental to shape competition and the incentives to enter a market.

Finally, macroeconomic applications of non-CES preferences with imperfect competition are only in their infancy because CES aggregators are ubiquitous. So far, additive preferences have been only rarely used to express per-period utility in dynamic models, but Bilbiie, Ghironi, and Melitz (2012) have recently investigated the case of homothetic preferences in a fully fledged dynamic model of business cycle. In principle, more general microfoundations of macroeconomic models could deliver a more realistic pattern for markups and business creation in both the short run and long run, but such an attempt still lies ahead (see Etro, 2016, building on our framework in a Ramsey model).

\section{Appendix A: Properties of symmetric functions}

Let us consider a twice-differentiable function $y=f(\mathbf{x})$ :

$$
f: \mathbb{R}_{+}^{n} \longrightarrow \mathbb{R} .
$$

We say that $f$ is symmetric with respect to its arguments $\mathbf{x}$ if

$$
f(\mathbf{x})=f(\mathbf{P x}), \quad \forall \mathbf{x}, \forall \mathbf{P},
$$

where $\mathbf{P}$ is a permutation matrix. The symmetry of $f$ imposes some symmetry also on its derivatives. Define:

$$
f_{i}(\mathbf{x})=\frac{\partial f(\mathbf{x})}{\partial x_{i}} \quad \text { and } \quad f_{i j}(\mathbf{x})=\frac{\partial^{2} f(\mathbf{x})}{\partial x_{i} \partial x_{j}},
$$

$i, j=1, \ldots, n$. Differentiation of (A1) with respect to $\mathbf{x}$ yields, in matrix notation:

$$
D f(\mathbf{x})=\mathbf{P}^{\prime} D f(\mathbf{P x}) .
$$

Because $\mathbf{P}$ is orthogonal, that is, $\mathbf{P}^{\prime}=\mathbf{P}^{-1}$, we obtain:

$$
\mathbf{P} D f(\mathbf{x})=D f(\mathbf{P x}), \quad \forall \mathbf{x}, \forall \mathbf{P},
$$

which shows that the gradient of a symmetric scalar function is itself a symmetric vector function. Similarly, differentiating (A2) with respect to $\mathbf{x}$ yields:

$$
\mathbf{P} D^{2} f(\mathbf{x})=D^{2} f(\mathbf{P x}) \mathbf{P}
$$

that is,

$$
\mathbf{P} D^{2} f(\mathbf{x}) \mathbf{P}^{\prime}=D^{2} f(\mathbf{P x}), \quad \forall \mathbf{x}, \forall \mathbf{P}
$$

(A2) and (A3) imply that:

$$
f_{i}(\mathbf{x})=\tilde{h}\left(x_{i}, \mathbf{x}_{-i}\right),
$$

where $\widetilde{h}$, which does not depend on $i$, is symmetric with respect to $\mathbf{x}_{-i}$. 
Indeed, it can be also proved that

$$
f_{i}(\mathbf{x})=h\left(x_{i}, \mathbf{x}\right),
$$

where $h$ is symmetric with respect to $\mathbf{x}$. The formal argument for this additional result was kindly provided by Giuseppe Savaré, of the Department of Mathematics of Pavia University. The proof follows. First, for each symmetric function $f(\mathbf{x})$ it holds that

$$
f(\mathbf{x})=\widetilde{f}\left(\mathbf{S}^{n}(\mathbf{x})\right),
$$

where $\widetilde{f}$ is unique and well defined and

$$
S_{m}^{n}(\mathbf{x})=\sum_{0<i_{1}<\ldots<i_{m} \leq n} \prod_{k=1}^{m} x_{i_{k}},
$$

with $m=1, \ldots, n$. This is so because any $\mathbf{x}$ is uniquely determined by the vector function $\mathbf{S}^{n}(\mathbf{x})$ (up to a permutation). Notice that the $n$ functions $S_{m}^{n}$ :

$$
\begin{aligned}
S_{1}^{n}(\mathbf{x})= & x_{1}+x_{2}+\cdots+x_{n}=\sum_{k=1}^{n} x_{k}, \\
S_{2}^{n}(\mathbf{x})= & x_{1} x_{2}+x_{1} x_{3}+\cdots+x_{1} x_{n}+\cdots x_{n-1} x_{n}=\sum_{0<i_{1}<i_{2} \leq n} x_{i_{1}} x_{i_{2}}, \\
S_{3}^{n}(\mathbf{x})= & \sum_{0<i_{1}<i_{2}<i_{3} \leq n} x_{i_{1}} x_{i_{2}} x_{i_{3}}, \\
& \ldots \\
S_{n}^{n}(\mathbf{x})= & x_{1} x_{2} \ldots x_{n}=\prod_{k=1}^{n} x_{k}
\end{aligned}
$$

are symmetric. Second, it turns out that:

$$
S_{k}^{n-1}\left(\mathbf{x}_{-i}\right)=\sum_{j=0}^{k}(-1)^{j} x_{i}^{j} S_{k-j}^{n}(\mathbf{x}),
$$

where $S_{0}^{n}=1$ : that is, $\mathbf{S}^{n-1}\left(\mathbf{x}_{-i}\right)$ can actually be written in terms of $\mathbf{S}^{n}(\mathbf{x})$. It follows immediately that:

$$
\widetilde{h}\left(x_{i}, \mathbf{x}_{-i}\right)=\widehat{h}\left(x_{i}, \mathbf{S}^{n-1}\left(\mathbf{x}_{-i}\right)\right)=h\left(x_{i}, \mathbf{x}\right),
$$

where $h$ is symmetric with respect to $\mathbf{x}$. These results imply Lemma 1.

\section{Appendix B: Endogenous Preferences}

Let $U$ and $V$ represent the preferences over the $N$ varieties, and suppose that only $n$ of them are available because the corresponding producers have entered the market. Then:

$$
U^{n}(\mathbf{x})=U(\mathbf{x}, 0 \iota) \quad \text { and } \quad V^{n}(\mathbf{s})=V\left(\mathbf{s}, s_{o}(\mathbf{s}) \iota\right),
$$

where $\iota$ is the unit vector of dimension $N-n$ and $s_{o}(\mathbf{s})$ is the (finite or infinite) choke price of the unavailable varieties, which possibly depends on the price of those actually provided. The derivation of the endogenous utility functions is immediate when preferences are additive, but not otherwise. Feenstra (2003) has analyzed the case of translog preferences. Here, we analyze two more examples used in the text.

Generalized Leontief preferences. The so-called generalized Leontief homothetic expenditure function (Diewert, 1971) corresponding to (22) is given by:

$$
E(\mathbf{p}, U)=U \sum_{i=1}^{N} \sum_{j=1}^{N} b_{i j} \sqrt{p_{i} p_{j}}=U \sqrt{\mathbf{p}} \mathbf{B} \sqrt{\mathbf{p}},
$$

where $\sqrt{\mathbf{p}^{\prime}}=\left[\sqrt{p_{1}}, \ldots, \sqrt{p_{N}}\right]$ is the (row) vector of square roots of the elements of $\mathbf{p}$, and $N$ is the total number of potential varieties, to be kept constant. The demand system is given by:

$$
\begin{aligned}
\mathbf{x}(\mathbf{p}, U) & =D_{\mathbf{p}} E(\mathbf{p}, U)=U(\widehat{\sqrt{\mathbf{p}}})^{-1} \mathbf{B} \sqrt{\mathbf{p}}, \\
x_{i}(\mathbf{p}, U) & =U\left[b+\sum_{j \neq i} \sqrt{\frac{p_{j}}{p_{i}}}\right],
\end{aligned}
$$




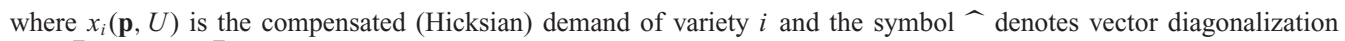
$\left(\widehat{\mathbf{z}}=\left[\begin{array}{ccc}z_{1} & 0 & 0 \\ 0 & \ddots & 0 \\ 0 & 0 & z_{N}\end{array}\right]\right)$. Notice that $b$ does not enter the Jacobian $D_{\mathbf{p}} x(\mathbf{p}, U)$, and thus the regularity conditions it has to satisfy (negative semidefiniteness). However, if all prices are the same, $x_{i}(p \boldsymbol{\iota}, U)=U[b+(N-1)]$, which gives the condition $N>1-b$.

Finally, the value of $b$ is crucial to account for some variety not being available for consumption because of being too expensive. Using (B2), one can write (in matrix form) $E(\mathbf{p}, U)=\mathbf{p}^{\prime} \mathbf{x}(\mathbf{p}, U)$, as it has to be by homogeneity and Shephard's Lemma. Now, let us partition the varieties into the set $I$ of varieties actually consumed, $i=1,2, \ldots, n$, and the set $J$ of varieties not available, $j=n+1, n+2, \ldots, N$, with $\mathbf{p}^{I}=\left[p_{1}, \ldots p_{n}\right], \mathbf{p}^{J}=\left[p_{n+1}, \ldots p_{N}\right], \mathbf{B}=\left[\begin{array}{ll}\mathbf{B}^{I I} & \mathbf{B}^{I J} \\ \mathbf{B}^{J I} & \mathbf{B}^{J J}\end{array}\right]$, and similarly. By (B2):

$$
\mathbf{x}^{J}=U\left(\widehat{\sqrt{\mathbf{p}^{J}}}\right)^{-1}\left[\mathbf{B}^{J I} \quad \mathbf{B}^{J J}\right] \sqrt{\mathbf{p}}=[\mathbf{0}]
$$

and it must be the case that

$$
\sqrt{\mathbf{p}^{J}}=-\left(\mathbf{B}^{J J}\right)^{-1} \mathbf{B}^{J I} \sqrt{\mathbf{p}^{I}}
$$

if $\mathbf{B}^{J J}$ is invertible. However, $\mathbf{B}^{J J}$ can be written as

$$
\mathbf{B}^{J J}=(1-b)\left[\frac{1}{1-b} \mathbf{1}_{N-n, N-n}-\mathbf{I}_{N-n, N-n}\right]
$$

where $\mathbf{1}_{N-n, N-n}$ is a $(N-n, N-n)$ matrix with unit entries and $\mathbf{I}$ is the relevant identity matrix. Because $\mathbf{1}_{N-n, N-n}$ has eigenvalues $(N-n)$ and zero, $\mathbf{B}^{J J}$ has eigenvalues $N-n-1+b$ and $b-1$, and it will then be invertible if $N-n-1 \neq-b \neq-1$. Because

$$
\begin{aligned}
& \mathbf{x}^{I}=U\left(\widehat{\sqrt{\mathbf{p}^{I}}}\right)^{-1}\left[\begin{array}{ll}
\mathbf{B}^{I I} & \mathbf{B}^{I J}
\end{array}\right]\left[\begin{array}{c}
\sqrt{\mathbf{p}^{I}} \\
\sqrt{\mathbf{p}^{J}}
\end{array}\right] \\
& =U\left(\widehat{\sqrt{\mathbf{p}^{I}}}\right)^{-1}\left[\begin{array}{ll}
\mathbf{B}^{I I} & \mathbf{B}^{I J}
\end{array}\right]\left[\begin{array}{c}
\sqrt{\mathbf{p}^{I}} \\
-\left(\mathbf{B}^{J J}\right)^{-1} \mathbf{B}^{J I} \sqrt{\mathbf{p}^{I}}
\end{array}\right],
\end{aligned}
$$

we obtain:

$$
\begin{aligned}
& E^{n}\left(\mathbf{p}, U^{n}\right)=\mathbf{p}^{I \prime} \mathbf{x}^{I}=U^{n} \mathbf{p}^{I \prime}\left(\widehat{\sqrt{\mathbf{p}^{I}}}\right)^{-1}\left[\begin{array}{ll}
\mathbf{B}^{I I} & \mathbf{B}^{I J}
\end{array}\right]\left[\begin{array}{c}
\sqrt{\mathbf{p}^{I}} \\
-\left(\mathbf{B}^{J J}\right)^{-1} \mathbf{B}^{J I} \sqrt{\mathbf{p}^{I}}
\end{array}\right] \\
& =U^{n}{\sqrt{\mathbf{p}^{I}}}^{\prime}\left[\begin{array}{ll}
\mathbf{B}^{I I} & \mathbf{B}^{I J}
\end{array}\right]\left[\begin{array}{c}
\sqrt{\mathbf{p}^{I}} \\
-\left(\mathbf{B}^{J J}\right)^{-1} \mathbf{B}^{J I} \sqrt{\mathbf{p}^{I}}
\end{array}\right] \\
& =U^{n}\left[\sqrt{\mathbf{p}^{I}} \mathbf{B}^{I I} \sqrt{\mathbf{p}^{I}}-\sqrt{\mathbf{p}^{I}} \mathbf{B}^{I J}\left(\mathbf{B}^{J J}\right)^{-1} \mathbf{B}^{J I} \sqrt{\mathbf{p}^{I}}\right] \\
& =U^{n}{\sqrt{\mathbf{p}^{I}}}^{\prime}\left[\mathbf{B}^{I I}-\mathbf{B}^{I J}\left(\mathbf{B}^{J J}\right)^{-1} \mathbf{B}^{J I}\right] \sqrt{\mathbf{p}^{I}} \\
& =U^{n}{\sqrt{\mathbf{p}^{I}}}^{\prime} \mathbf{A} \sqrt{\mathbf{p}^{I}},
\end{aligned}
$$

which delivers the indirect utility (23) with $\mathbf{A} \equiv\left[\mathbf{B}^{I I}-\mathbf{B}^{I J}\left(\mathbf{B}^{J J}\right)^{-1} \mathbf{B}^{J I}\right]$ as the symmetric $(n, n)$ matrix whose elements will be discussed below.

Notice that $\mathbf{B}^{I J}=\mathbf{B}^{J I}=\mathbf{1}_{n, N-n}$, and that $\mathbf{B}^{J J}$ is a $(N-n, N-n)$ square matrix whose diagonal elements are equal to $b$ and extradiagonal elements are equal to 1 . Because:

$$
\mathbf{B}^{J J} \mathbf{1}_{N-n}=[(N-n)-1+b] \mathbf{1}_{N-n}=\lambda \mathbf{1}_{N-n}
$$

has the obvious solution $\lambda=N-n-1+b$, then $\mathbf{B}^{J J}$ has a unit eigenvector associated to the eigenvalue $N-n-1+b$. Accordingly, its inverse has a unit eigenvector associated to an eigenvalue given by $(N-n-1+b)^{-1}$. From this, we can compute:

$$
\left(\mathbf{B}^{J J}\right)^{-1} \mathbf{B}^{J I}=\frac{1}{N-n-1+b} \mathbf{1}_{N-n, n}
$$

and

$$
\mathbf{B}^{I J}\left(\mathbf{B}^{J J}\right)^{-1} \mathbf{B}^{J I}=\frac{N-n}{N-n-1+b} \mathbf{1}_{n, n} .
$$


Notice that replacing the first expression in (B3) implies $\sqrt{\mathbf{p}^{J}}=-[N-n-1+b]^{-1} \boldsymbol{\iota}^{\prime} \sqrt{\mathbf{p}^{I}}$, which uniquely identifies the choke price:

$$
p_{0}=\left(\frac{\sum_{j=1}^{n} \sqrt{p_{j}}}{N-n-1+b}\right)^{2}
$$

Because $\mathbf{B}^{I I}=\left[\mathbf{1}_{n, n}-(1-b) \mathbf{I}_{n, n}\right]$, it follows that:

$$
\begin{aligned}
\mathbf{A} & =\mathbf{B}^{I I}-\mathbf{B}^{I J}\left(\mathbf{B}^{J J}\right)^{-1} \mathbf{B}^{J I}=\left[\mathbf{1}_{n, n}-(1-b) \mathbf{I}_{n, n}\right]-\frac{N-n}{N-n-1+b} \mathbf{1}_{n, n} \\
& =\frac{b-1}{N-n-1+b} \mathbf{1}_{n, n}-(1-b) \mathbf{I}_{n, n} .
\end{aligned}
$$

Accordingly,

$$
a_{i i}=\underline{a}=\frac{(b-1)(N-n+b)}{N-n-1+b}, \quad a_{i j}=a=\frac{b-1}{N-n-1+b}
$$

for $i, j=1, \ldots, n, i \neq j$. Notice that any $b \in(-N+1,-N+2)$ satisfies all our assumptions: in particular, this range implies that $b<0, N>1-b$, and that $\mathbf{B}^{J J}$ is a negative definite matrix. This ensures that $a>0>\underline{a}$, so that the regularity conditions for the utility function (23) are satisfied. As we discuss in the main text, it also guarantees that $\varepsilon^{n}>1$ for $n \geq 2$.

Quadratic indirect utility. Consider now the generalized quadratic indirect utility:

$$
V(\mathbf{s})=\mathbf{s}^{\prime} \boldsymbol{\beta} \mathbf{s}-\alpha S,
$$

where $S=\sum_{j=1}^{N} s_{j}$, and to achieve symmetry, it is assumed that $\beta_{i j}=\beta$ and $\beta_{i i}=\underline{\beta}$ for $i, j=1, \ldots, N, i \neq j$, and $N$ is the total number of potential varieties. Notice that:

$$
\boldsymbol{\beta}=\beta \mathbf{1}_{N, N}-(\beta-\underline{\beta}) \mathbf{I}=(\beta-\underline{\beta})\left[\frac{\beta}{(\beta-\underline{\beta})} \mathbf{1}_{N, N}-\mathbf{I}\right] .
$$

As $\mathbf{1}_{N, N}$ has eigenvalues $N$ and 0 , the eigenvalues of $\boldsymbol{\beta}$ are then given by $(N-1) \beta+\underline{\beta}$ and $\underline{\beta}-\beta$. Because:

$$
\begin{aligned}
D V(\mathbf{s}) & =2 \boldsymbol{\beta} \mathbf{s}-\alpha \boldsymbol{\iota} D^{2} V(\mathbf{s})=2 \boldsymbol{\beta}, \\
\frac{\partial V(s \boldsymbol{\imath})}{\partial s_{i}} & =2 \sum_{j=1}^{N} \beta_{i j} s-\alpha=2 s[\underline{\beta}+(N-1) \beta]-\alpha,
\end{aligned}
$$

to satisfy the conditions of monotonicity and quasiconvexity of $V$, we assume that $\alpha>0$, and that $\beta$ is a positive semidefinite matrix. In particular, we assume that $(N-1) \beta \geq-\underline{\beta}, \underline{\beta}>\beta, \underline{\beta}>0$ and restrict attention to the case in which $\alpha \iota>2 \beta$ s. We obtain:

$$
\mathbf{x}(\mathbf{s})=\frac{D V(\mathbf{s})}{D V(\mathbf{s})^{\prime} \mathbf{s}}=-\frac{\alpha \iota-2 \boldsymbol{\beta} \mathbf{s}}{2 \mathbf{s}^{\prime} \boldsymbol{\beta} \mathbf{s}-\alpha \iota^{\prime} \mathbf{s}}
$$

with

$$
V(s \iota)=N s\{s[(N-1) \beta+\underline{\beta}]-\alpha\}<0 .
$$

As in the previous section, let us partition the varieties into the set $I$ of varieties actually consumed, $i=1,2, \ldots, n$, and the set $J$ of varieties not available, $j=n+1, n+2, \ldots, N$, with $\mathbf{s}^{I}=\left[s_{1}, \ldots s_{n}\right], \mathbf{s}^{J}=\left[s_{n+1}, \ldots s_{N}\right]$, $\boldsymbol{\beta}=\left[\begin{array}{ll}\boldsymbol{\beta}^{I I} & \boldsymbol{\beta}^{I J} \\ \boldsymbol{\beta}^{J I} & \boldsymbol{\beta}^{J J}\end{array}\right]$, and similarly. Notice that, by (B6), $\mathbf{x}^{J}=[\mathbf{0}]$ requires $\alpha \boldsymbol{\iota}=2\left[\boldsymbol{\beta}^{J I} \boldsymbol{\beta}^{J J}\right] \mathbf{s}=2\left(\boldsymbol{\beta}^{J I} \mathbf{s}^{I}+\boldsymbol{\beta}^{J J} \mathbf{s}^{J}\right)$, implying:

$$
\mathbf{s}^{J}=\frac{\alpha}{2}\left(\boldsymbol{\beta}^{J J}\right)^{-1} \iota-\left(\boldsymbol{\beta}^{J J}\right)^{-1} \boldsymbol{\beta}^{J I} \mathbf{s}^{I},
$$

because $\boldsymbol{\beta}^{J J}=(\beta-\underline{\beta})\left[\frac{\beta}{(\beta-\underline{\beta})} \mathbf{1}_{N-n, N-n}-\mathbf{I}\right]$ is invertible (and positive definite) under our assumptions. Moreover:

$$
\boldsymbol{\beta}^{J J} \iota=(\beta-\underline{\beta})\left[\frac{\beta}{(\beta-\underline{\beta})}(N-n)-1\right] \iota,
$$

and thus $\boldsymbol{\beta}^{J J}$ has a unit eigenvector associated to the positive eigenvalue given by $\beta(N-n-1)+\underline{\beta}$. This implies that $\left(\boldsymbol{\beta}^{J J}\right)^{-1}$ has a unit eigenvector associated to the positive eigenvalue $1 /[\beta(N-n-1)+\beta]$. 
As $\boldsymbol{\beta}^{J I}=\beta \mathbf{1}_{N-n, n}$, defining $S^{I}=\sum_{j=1}^{n} s_{j}$, we get:

$$
\mathbf{s}^{J}=\left[\frac{\alpha}{2}-\beta S^{I}\right]\left(\boldsymbol{\beta}^{J J}\right)^{-1} \iota=\frac{\frac{\alpha}{2}-\beta S^{I}}{\beta(N-n-1)+\underline{\beta}} \iota>0,
$$

which identifies the (normalized) choke price of the $N-n$ unproduced varieties as a function of the (normalized) prices of the $n$ varieties actually offered in market:

$$
s_{0}=\frac{\frac{\alpha}{2}-\beta S^{I}}{\beta(N-n-1)+\underline{\beta}} .
$$

Using (B7) and $S^{J}=(N-n) s_{0}$ yields:

$$
\begin{aligned}
V= & \mathbf{s}^{\prime} \boldsymbol{\beta} \mathbf{s}-\alpha\left(S^{I}+S^{J}\right)=\left[\begin{array}{ll}
\mathbf{s}^{I \prime} & \mathbf{s}^{J \prime}
\end{array}\right]\left[\begin{array}{ll}
\boldsymbol{\beta}^{I I} & \boldsymbol{\beta}^{I J} \\
\boldsymbol{\beta}^{J I} & \boldsymbol{\beta}^{J J}
\end{array}\right]\left[\begin{array}{l}
\mathbf{s}^{I} \\
\mathbf{s}^{J}
\end{array}\right]-\alpha\left(S^{I}+S^{J}\right) \\
= & {\left[\begin{array}{ll}
\mathbf{s}^{I \prime} & \mathbf{s}^{J \prime}
\end{array}\right]\left[\begin{array}{c}
\boldsymbol{\beta}^{I I} \mathbf{s}^{I}+\boldsymbol{\beta}^{I J} \mathbf{s}^{J} \\
\boldsymbol{\beta}^{J I} \mathbf{s}^{I}+\boldsymbol{\beta}^{J J} \mathbf{s}^{J}
\end{array}\right]-\alpha\left(S^{I}+S^{J}\right) } \\
= & \mathbf{s}^{I \prime} \boldsymbol{\beta}^{I I} \mathbf{s}^{I}+\mathbf{s}^{I \prime} \boldsymbol{\beta}^{I J} \mathbf{s}^{J}+\mathbf{s}^{J \prime} \boldsymbol{\beta}^{J I} \mathbf{s}^{I}+\mathbf{s}^{J \prime} \boldsymbol{\beta}^{J J} \mathbf{s}^{J}-\alpha S^{I}-\alpha S^{J} \\
= & \mathbf{s}^{I \prime} \boldsymbol{\beta}^{I I} \mathbf{s}^{I}-\alpha S^{I}+2(N-n) \beta S^{I} \frac{\frac{\alpha}{2}-\beta S^{I}}{\beta(N-n-1)+\underline{\beta}} \\
& +(N-n) \frac{\left(\frac{\alpha}{2}-\beta S^{I}\right)^{2}}{\beta(N-n-1)+\underline{\beta}}-\alpha(N-n) \frac{\alpha}{\beta(N-n-1)+\underline{\beta}} \\
= & \mathbf{s}^{I \prime} \boldsymbol{\beta}^{I I} \mathbf{s}^{I}-\alpha S^{I}+\frac{(n-N)\left(\frac{\alpha}{2}-\beta S^{I}\right)^{2}}{\beta(N-n-1)+\underline{\beta}}
\end{aligned}
$$

or:

$$
V=\mathbf{s}^{I \prime} \boldsymbol{\beta}^{I I} \mathbf{s}^{I}+\frac{(n-N)\left(\frac{\alpha^{2}}{4}+\beta^{2}\left(S^{I}\right)^{2}\right)-\alpha S^{I}(\underline{\beta}-\beta)}{\beta(N-n-1)+\underline{\beta}} .
$$

Let us normalize $\beta=-b<0, \underline{\beta}=b(N-1)$ and write $\bar{s} \equiv S^{I} / n$ for the average normalized price of the available varieties. Then all our assumptions are satisfied and we reach the indirect utility:

$$
\begin{aligned}
V^{n} & =\sum_{i=1}^{n} \sum_{j=1}^{n} \beta_{i j} s_{i} s_{j}+\frac{N-n}{n \beta} \frac{\alpha^{2}}{4}+\frac{N-n}{n} \beta\left(S^{I}\right)^{2}-\frac{\alpha N S^{I}}{n} \\
& =\sum_{i=1}^{n} \sum_{j=1}^{n} \beta_{i j} s_{i} s_{j}-\frac{N-n}{n b} \frac{\alpha^{2}}{4}-n b(N-n) \bar{s}^{2}-\alpha N \bar{s},
\end{aligned}
$$

which endogenously depends on the number of provided varieties and their prices. Rearranging terms, we get (38).

\section{References}

Anderson, S., ERKAL, N., AND PiccinIn, D. “Aggregative Oligopoly Games with Entry.” Mimeo, University of Virginia, Charlottesville, 2012.

Arkolakis, C., Costinot, A., Donaldson, D., and Rodriguez-Clare, A. "The Elusive Pro-Competitive Effects of Trade.” Working Paper no. 21370, NBER, Cambridge, MA, 2015.

Behrens, K. ANd Murata, Y. “Trade, Competition, and Efficiency.” Journal of International Economics, Vol. 87 (2012), pp. 1-17.

Benassy, J.-P. “Taste for Variety and Optimum Production Patterns in Monopolistic Competition.” Economics Letters, Vol. 52 (1996), pp. 41-47.

Bertoletti, P. "Economic Integration Effects on Market Structure.” In S. Urban and C. Nanopoulos, eds., Information and Management. Wiesbaden: Gabler, 1998.

Bertoletti, P. And EPIfani, P. “Monopolistic Competition: CES Redux?” Journal of International Economics, Vol. 93(2) (2014), pp. 227-238.

Bertoletti, P. And Etro, F. "Pricing to Market in the Krugman Model.” Economics Bulletin, Vol. 34 (2014), pp. $459-468$. . "Monopolistic Competition When Income Matters.” Economic Journal, (2014).

Bertoletti, P., Etro, F., And Simonovska, I. "International Trade with Indirect Additivity.” Working Paper no. 21984, NBER, Cambridge, MA, 2016. 
Bertoletti, P., Fumagalli, E., And Poletti, C. "Price-Increasing Monopolistic Competition? The Case of IES Preferences.” Working Paper no. 15, IEFE, Bocconi University, Milan, 2008.

Bilbie, F., Ghironi, F., And Melitz, M. "Endogenous Entry, Product Variety, and Business Cycles.” Journal of Political Economy, Vol. 120 (2012), pp. 304-345.

Blackorby, C. AND Russell, R. "The Morishima Elasticity of Substitution: Symmetry, Constancy, Separability, and Its Relationship to the Hicks and Allen Elasticities.” Review of Economic Studies, Vol. 48 (1981), pp. 147-158.

- "Will the Real Elasticity of Substitution Plase Stand Up? (A Comparison of the Allen/Uzawa and Morishima Elasticities)." American Economic Review, Vol. 79 (1989), pp. 882-888.

Campbell, J. and Hopenhayn, H. "Market Size Matters." Journal of Industrial Economics, Vol. 53 (2005), pp. 1-25.

Chen, Y. And Riordan, M. "Price and Variety in the Spokes Model." Economic Journal, Vol. 117 (2007), pp. 897-921.

Dasgupta, P. And Stiglitz, J. "Industrial Structure and the Nature of Innovative Activity." Economic Journal, Vol. 90 (1980), pp. 266-293.

D’aspremont, C., Dos Santos Ferreira, R., and Gerard-Varet, L.-A. "Competition for Market Share or for Market Size: Oligopolistic Equilibria with Varying Competitive Toughness.” International Economic Review, Vol. 48 (2007), pp. 761-784.

Di Comite, F., Thisse, J.F., And Vandenbussche, H. "Verti-zontal Differentiation in Export Markets." Journal of International Economics, Vol. 93 (2014), pp. 50-66.

Diewert, W. “An Application of the Shephard Duality Theorem: A Generalized Leontief Production Function.” Journal of Political Economy, Vol. 79 (1971), pp. 481-507.

Dixit, A. And Stiglitz, J. "Monopolistic Competition and Optimum Product Diversity." American Economic Review, Vol. 67 (1977), pp. 297-308.

ETRO, F. “Aggressive Leaders.” RAND Journal of Economics, Vol. 37 (2006), pp. 146-154.

. "Endogenous Market Structures and International Trade." Scandinavian Journal of Economics, Vol. 117 (2015), pp. 918-956.

. "The Ramsey Model with Monopolistic Competition and General Preferences." Economics Letters, Vol. 145 (2016), pp. 141-144.

Etro, F. And Colciago, A. "Endogenous Market Structures and the Business Cycle.” Economic Journal, Vol. 120 (2010), pp. 1201-1234.

Feenstra, R. "A Homothetic Utility Function for Monopolistic Competition Models without Constant Price Elasticity." Economics Letters, Vol. 78 (2003), pp. 79-86.

- "Restoring the Product Variety and Pro-Competitive Gains from Trade with Heterogeneous Firms and Bounded Productivity." Working Paper no. 16796, NBER, Cambridge, MA, 2014.

HANoch, G. "Production and Demand Models with Direct or Indirect Implicit Additivity." Econometrica, Vol. 43 (1974), pp. 395-419.

Hicks, J. "Elasticities of Substitution Again: Substitutes and Complements." Oxford Economic Papers, Vol. 22 (1970), pp. 289-296.

HouthakKer, H.S. “Additive Preferences.” Econometrica, Vol. 28 (1960), pp. 244-257.

Kimball, M.S. "The Quantitative Analytics of the Basic Neomonetarist Model." Journal of Money, Credit and Banking, Vol. 27 (1995), pp. 1241-1277.

Krugman, P. "Increasing Returns, Monopolistic Competition, and International Trade." Journal of International Economics, Vol. 9 (1979), pp. 469-479.

—. "Scale Economies, Product Differentiation, and the Pattern of Trade." American Economic Review, Vol. 70 (1980), pp. 950-959.

MeLitz, M. "The Impact of Trade on Intra-Industry Reallocations and Aggregate Industry Productivity." Econometrica, Vol. 71 (2003), pp. 1695-1725.

Melitz, M. and Ottaviano, G. "Market Size, Trade, and Productivity.” Review of Economic Studies, Vol. 75 (2008), pp. 295-316.

Mrázová, M. And Neary, P. "Together at Last: Trade Costs, Demand Structure, and Welfare." American Economic Review, Vol. 104 (2014), pp. 298-303.

Nekarda, C. And Ramey, V. "The Cyclical Behavior of the Price-Cost Markup." Working Paper no. 19099, NBER, Cambridge, MA, 2013.

Nocco, A., Ottaviano, G., and Salto, M. "Monopolistic Competition and Optimum Product Selection." American Economic Review, Vol. 104 (2014), pp. 304-309.

Parenti, M., Ushchev, P., And Thisse, J.-F. “Toward a Theory of Monopolistic Competition.” Working Paper no. 10014, CEPR, London, 2014.

Pollak, R. “Generalized Separability.” Econometrica, Vol. 40 (1972), pp. 431-453.

Romer, P. “Endogenous Technological Change.” Journal of Political Economy, Vol. 98 (1990), pp. S71-S102.

Simonovska, I. "Income Differences and Prices of Tradables.” Review of Economic Studies, Vol. 82 (2015), pp. 16121656.

Spence, A.M. "Product Selection, Fixed Costs, and Monopolistic Competition." Review of Economic Studies, Vol. 43 (1976), pp. 217-235. 
Sutton, J. Sunk Costs and Market Structure: Price Competition, Advertising, and the Evolution of Concentration. London: MIT Press, 1991.

VARIAn, H. Microeconomic Analysis. New York: Norton \& Company, 1992.

Vives, X. "On the Efficiency of Bertrand and Cournot Equilibria with Product Differentation." Journal of Economic Theory, Vol. 36 (1985), pp. 166-175.

. Oligopoly Pricing. Old Ideas and New Tools. London: MIT Press, 1999.

"Innovation and Competitive Pressure." Journal of Industrial Economics, Vol. 56 (2008), pp. 419-469.

YANG, X. AND HeIJDRA, B.J. "Monopolistic Competition and Optimum Product Diversity: Comment.” American Economic Review, Vol. 83 (1993), pp. 295-301.

Zhelobodko, E., Kokovin, S., Parenti, M., And Thisse, J.-F. "Monopolistic Competition in General Equilibrium: Beyond the CES.” Econometrica, Vol. 80 (2012), pp. 2765-2784. 